\title{
基于原子干涉技术的精密重力测量研究
}

\author{
陈乐乐, 罗覃, 邓小兵, 谈玉杰, 毛德凯, 张恒, 周敏康, 段小春, 邵成刚, 胡忠坤* \\ 华中科技大学物理学院, 基本物理量测量教育部重点实验室, 武汉 430074 \\ *联系人, E-mail: zkhu@hust.edu.cn
}

收稿日期: 2016-03-16; 接受日期: 2016-04-25; 网络出版日期: 2016-06-15

国家自然科学基金资助项目(编号：41127002, 11204094, 11205064, 11474115)

\begin{abstract}
摘要基于双光子受激拉曼跃迁的冷原子干涉仪, 在基础物理研究和精密测量应用方面倍受关注, 其中精密 重力测量是原子干涉仪的重要应用之一. 本文描述了原子干涉重力测量的原理, 包括原子波包在重力场中的自 由演化、激光与原子相互作用以及原子物质波的干涉过程; 介绍了原子重力仪的典型实验设计、测量过程和 发展趋势; 综述了国内外小组在原子干涉重力测量方面的研究工作. 此外本文还对当前原子干涉重力仪的主要 噪声及系统误差进行了分析, 介绍了背景振动、拉曼光相位噪声的抑制技术以及主要系统误差效应的评估方 法. 最后, 本文还介绍了原子干涉测量技术在万有引力常数测量以及弱等效原理检验等基础物理研究中的应用.
\end{abstract}

关键词物质波, 重力加速度, 原子干涉仪, 冷原子

PACS: 03.75.-b, 06.30.Gv, 07.07.Df, 39.25.+k

\section{1 背景介绍}

\section{1 精密重力测量}

精密重力测量在引力基础科学问题研究和地球 物理应用研究中有着广泛应用. 在基础物理研究方面, 精密重力测量可用于质量基准的建立 ${ }^{[1]}$ 以及后牛顿引 力理论的检验 ${ }^{[2,3]}$. 在地球物理中, 高精度重力数据可 用于地球动力学研究以及潮汐模型的建立 ${ }^{[4]}$. 在资源 勘探中, 重力及重力梯度数据可用于重力反演来得到 地下的质量分布, 从而为资源勘探提供重要线索 ${ }^{[5]}$.

重力仪是测量重力加速度的仪器, 它可分为绝对 重力仪以及相对重力仪两种. 相对重力仪能够灵敏地 测量出重力加速度大小的相对变化. 绝对重力仪能够
精确地测量出重力加速度的绝对大小. 目前商用化程 度最高的绝对重力仪是FG-5(X)型角雉下落式的绝对 重力仪, 其 $1 \mathrm{~s}$ 积分时间内的测量分辨率约 $15 \mu \mathrm{Gal}(1$ $\mu \mathrm{Gal}=1 \times 10^{-8} \mathrm{~m} / \mathrm{s}^{2}$ ), 测量不确定度为 $2-5 \mu \mathrm{Gal}^{[6,7]}$, 该重 力仪主要受到机械磨损等因素的限制. 20 世纪末期发 展起来的原子干涉测量技术, 不存在机械磨损的问题, 工作频率可以达到 $1 \mathrm{~Hz}$ 量级. 原子干涉技术用于重力 测量以后, 随着相关技术的改进, 分辨率最高的结果 ${ }^{[8]}$ 已达到 $4.2 \mu \mathrm{Gal} / \sqrt{\mathrm{Hz}}$, 其不确定度水平也与 $\mathrm{FG}-5$ 相当.

\section{2 原子干涉重力测量}

20 世纪 80 年代以后, 随着中性原子的激光冷却技 术的发展 ${ }^{[9-13]}$, 冷原子干涉仪应运而生. 1991年, 斯坦

引用格式: 陈乐乐, 罗覃, 邓小兵, 等. 基于原子干涉技术的精密重力测量研究. 中国科学: 物理学力学天文学, 2016, 46: 073003 Chen L L, Luo Q, Deng X B, et al. Precision gravity measurements with cold atom interferometer (in Chinese). Sci Sin-Phys Mech Astron, 2016, 46: 073003, doi: 10.1360/SSPMA2016-00156 
福大学Kasevich等人 ${ }^{[14]}$ 将冷原子干涉技术用于重力 加速度的测量, 利用钠原子干涉仪首次测量的结果 在积分 $1000 \mathrm{~s}$ 之后的分辨到 $3 \mathrm{mGal}$ 的水平, 改进后积 分 $2000 \mathrm{~s}$ 可以分辨到 $30 \mu \mathrm{Gal}$. 此后, 该小组用铯原子干 涉仪进行重力测量, 测量不确定度达到 $3.4 \mu \mathrm{Ga}^{[}{ }^{[15]}$. 由 于原子干涉重力测量具有很高的潜在分辨率, 国际上 各个小组也开始了对原子干涉重力测量的研究, 包括 法国巴黎天文台、国际计量局、德国汉诺威大学、 德国柏林洪堡大学, 国内中国科学院武汉物理与数学 研究所、浙江大学、华中科技大学等也相继开展了 研究.

根据原子干涉的原理 ${ }^{[16]}$, 原子干涉重力仪可分为 两大类:

(1) 自由下落式的原子干涉重力仪一一这种类型 的原子干涉重力仪利用双光子受激拉曼跃迁对原子 进行操控. 如美国斯坦福大学朱棣文小组的铯原子喷 泉原子重力仪 ${ }^{[15]}$ 、德国柏林洪堡大学的铭原子喷泉

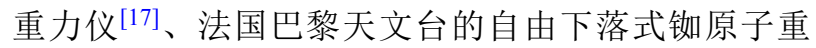
力仪 ${ }^{[18]}$ 以及国内的中国科学院武汉物理与数学研究

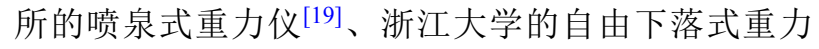
仪 ${ }^{[20]}$ 和华中科技大学铭原子喷泉重力仪 ${ }^{[8,21]}$ 等都属于 此类重力仪, 其中部分装置如图 $1^{[22,23]}$ 所示.

(2) 囚禁式的原子干涉重力仪一一如法国巴黎天 文台 ${ }^{[24]}$ 和意大利佛罗伦萨大学的光晶格重力仪 ${ }^{[25-27]}$. 在光晶格原子干涉重力仪中, 原子被囚禁在光晶格中, 通过测量布洛赫振荡频率来测量重力加速度. 目前, 这种囚禁式的原子重力仪分辨率低于自由下落式的
原子重力仪, 但在测量短程相互作用时更有优势.

\section{3 原子干涉技术在精密测量中的应用}

原子干涉技术利用被激光冷却以后的超冷原子 团作为检验质量, 冷原子团在整个干涉过程中除了动 量态发生改变外还存在内态改变, 由此能够实现形式 复杂多样的干涉仪类型, 也为精密测量提供了更多的 测量方案.

原子干涉仪在精密测量的应用中, 除了用于测 量重力加速度外, 利用萨奈克效应可用于测量地球 转动 ${ }^{[28-32]}$; 通过上抛不同高度的原子, 原子干涉仪可 用于测量坚直方向的重力梯度 ${ }^{[33-37]}$; 通过改变干涉 仪附近的质量分布配置, 可以用于测量万有引力常 数 $G^{[38-40]}$. 此外可以将原子制备在磁敏感态来精密 测量磁场梯度 ${ }^{[41,42]}$; 在同一个干涉区间实现不同种类 原子或原子不同内态的重力测量, 用于检验弱等效 原理 ${ }^{[43]}$. 在其他应用中, 原子干涉仪还可以用于测量 精细结构常数 ${ }^{[44,45]}$, 探测引力波 ${ }^{[46-48]}$. 太空原子干涉 重力测量也在发展中 ${ }^{[49,50]}$.

\section{2 原子干涉重力测量原理及实验}

\section{1 原子干涉重力测量原理}

\subsection{1 物质波在梯度重力场中的自由演化}

本文在此采用 $A B C D$ 矩阵 ${ }^{[51]}$ 的方法计算原子在重 力梯度场中的自由演化. 在梯度重力场中，原子自由

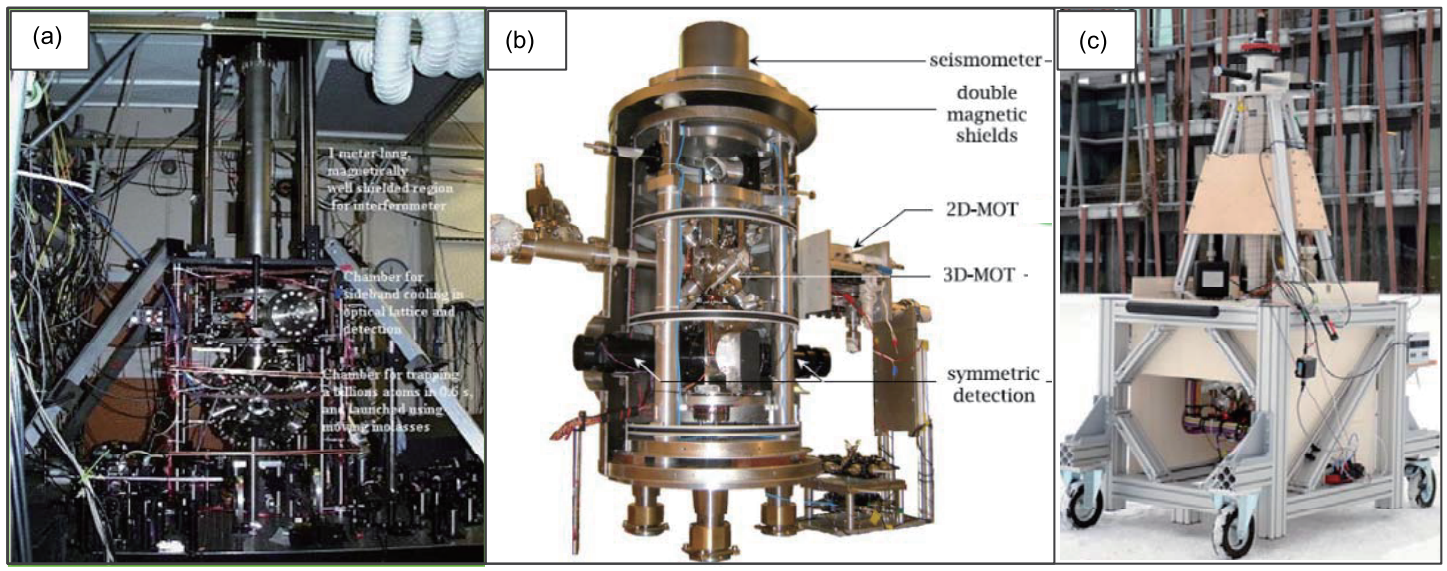

图1 (网络版彩图)原子干涉重力仪的实物图. (a) 斯坦福大学的原子干涉重力仪; (b) 巴黎天文台的原子干涉重力仪 ${ }^{[22]}$; (c) 柏林洪堡大学的原子干涉重力仪 ${ }^{[23]}$

Figure 1 (Color online) The apparatus of atom gravimeters at: (a) Stanford University, (b) SYRTE [22] and (c) Berlin Humboldt University [23]. 
演化时, 整个系统的哈密顿量形式为

$H=H_{0}+\frac{p_{\mathrm{op}}^{2}}{2 M}+M\left(g-\gamma z_{\mathrm{op}} / 2\right) z_{\mathrm{op}}+V\left(r_{\mathrm{op}}, t\right)$,

其中 $\gamma$ 为重力梯度, $H_{0}$ 为原子态的能量大小, $V\left(r_{\mathrm{op}}, t\right)$ 对 应其他物理场的能量. $p_{\mathrm{op}}$ 为动量算符, $z_{\mathrm{op}}$ 为位移算符, $M$ 为原子的质量大小. 假设原子的波包是高斯型的, 波 包的形式可表示为

$\psi_{t_{0}}=\frac{1}{\sqrt{X_{0}}} \exp \left[\frac{\mathrm{i} M}{2 \hbar} \frac{Y_{0}}{X_{0}}\left(z-z_{0}\right)^{2}\right] \exp \left[\mathrm{i} M v_{0}\left(z-z_{0}\right) / \hbar\right]$,

其中 $z_{0}$ 为原子的中心位置, $v_{0}$ 为初始速度, $X_{0}, Y_{0}$ 是原 子波包在相空间中的宽度. 原子波包在梯度重力场中 的演化过程满足

$$
\begin{aligned}
\psi(z, t) & =\int_{-\infty}^{\infty} \mathrm{d} z^{\prime} K\left(z, z^{\prime}, t, t_{0}\right) \psi_{t_{0}} \\
& =\exp \left[\frac{\mathrm{i}}{\hbar} S_{\mathrm{cl}}\right] \psi_{t_{0}}\left(z-z_{\mathrm{cl}}, v_{\mathrm{cl}}, X, Y\right),
\end{aligned}
$$

其中 $z_{\mathrm{cl}}$ 为经典位移, $v_{\mathrm{cl}}$ 为经典速度. 经典作用量

$$
\begin{aligned}
S_{\mathrm{cl}}= & -\frac{M}{2} \int_{t_{0}}^{t}\left(\dot{\xi}^{2}-\gamma \xi^{2}\right) \mathrm{d} t_{1}+M \dot{\xi} \xi \\
& +m \dot{\xi}\left(A z_{0}+B v_{0}\right)+\frac{M}{2}\left(A C z_{0}^{2}+D B v_{0}^{2}+2 B C z_{0} v_{0}\right),
\end{aligned}
$$

其中，

$$
\begin{array}{ll}
A=\cosh (\sqrt{\gamma} t), & B=\frac{1}{\sqrt{\gamma}} \sinh (\sqrt{\gamma} t), \\
C=\sqrt{\gamma} \sinh (\sqrt{\gamma t}), & D=\cosh (\sqrt{\gamma t}) .
\end{array}
$$

$\xi$ 是与原子的初始位移及速度相关的物理量, 且 满足方程

$\ddot{\xi}-\gamma \xi+g=0, L-M\left(\frac{1}{2} \dot{\xi}^{2}+\frac{\gamma}{2} \xi^{2}-g r\right)=0$,

$L$ 为系统的拉氏量.

经典位置以及经典速度的改变满足下列关系:

$z_{\mathrm{cl}}=A z_{0}+B v_{0}+\xi, \quad X=A X_{0}+B Y_{0}$,

$v_{\mathrm{cl}}=C z_{0}+D v_{0}+\dot{\xi}, \quad Y=C X_{0}+D Y_{0}$.

\subsection{2 激光与原子相互作用}

相比于自由演化, 激光与原子相互作用时需考虑 原子的不同能级的能级差以及激光与原子相互作用 强度. 由于激光与原子作用时间较短, 在激光与原子 相互作用时可忽略重力变化. 拉曼光与三能级原子相
互作用(图2所示)在失谐量 $\Delta$ 远大于 $\delta$ 的情况下能够简 化为与二能级相互作用模型来处理 ${ }^{[52]}$. 相应地激光与 原子相互作用时波函数以及哈密顿量都应写成矩阵 的形式

$\boldsymbol{\Psi}(\boldsymbol{r}, t)=\left[\begin{array}{l}\Psi_{b}(z, t) \\ \Psi_{a}(z, t)\end{array}\right]$,

$\boldsymbol{H}_{0}=\left[\begin{array}{cc}E_{b} & 0 \\ 0 & E_{a}\end{array}\right]$,

$\boldsymbol{V}(\boldsymbol{r}, t)$
$=\left[\begin{array}{ll}V_{b b} & -\hbar \Omega_{b a} U^{*}(r, t) \exp \left[-\mathrm{i}\left(\omega_{\mathrm{eff}} t-k_{\mathrm{eff}} z+\phi_{0}\right)\right. \\ -\hbar \Omega_{a b} U(\boldsymbol{r}, t) \exp \left[\mathrm{i}\left(\omega_{\mathrm{eff}} t-k_{\mathrm{eff}} \boldsymbol{\phi}_{0}\right)\right] & V_{a a}\end{array}\right]$,

其中 $E_{b}, E_{a}$ 为原子对应能级的能量, $\Omega_{b a}, \Omega_{a b}$ 为激光与 原子作用的等效拉比频率, 它们与激光失谐量 $\Delta, \delta$ 及 光强相关, $k_{\mathrm{eff}}$ 为拉曼光的等效波矢, $\omega_{\mathrm{eff}}$ 为拉曼光的等 效频率. $\phi_{0}$ 是拉曼光的等效初相位. 在光场强度为常 数 $(U(\boldsymbol{r}, t) \equiv 1)$, 失谐量 $\delta \approx 0$ 时, 激光脉冲对应的跃迁 矩阵为

$\left[\begin{array}{cc}\cos \left(\frac{\Omega \tau}{2}\right) & -\mathrm{i} \sin \left(\frac{\Omega_{r} \tau}{2}\right) \mathrm{e}^{-\mathrm{i} \phi_{\text {eff }}} \\ -\mathrm{i} \sin \left(\frac{\Omega_{r} \tau}{2}\right) \mathrm{e}^{\mathrm{i} \phi_{\text {eff }}} & \cos \left(\frac{\Omega \tau}{2}\right)\end{array}\right]$,

其中 $\phi_{\mathrm{eff}}=k_{\mathrm{eff}} z-\omega_{\mathrm{eff}} t-\phi_{0}$ 是激光的等效相位. $\Omega \tau=\pi / 2$ 时激光脉冲为 $\pi / 2$ 脉冲, $\Omega \tau=\pi$ 时对应的激光脉冲为 $\pi$ 脉 冲. 发生内态的跃迁时, 原子将记录下激光与原子相 互作用的相位 $\phi_{\mathrm{eff}}$.

\subsection{3 原子干涉仪相位}

在均匀重力场中, 原子走不同路径(Arm I, Arm II) 的轨迹是闭合的. 闭合路径对应原子自由演化的相位

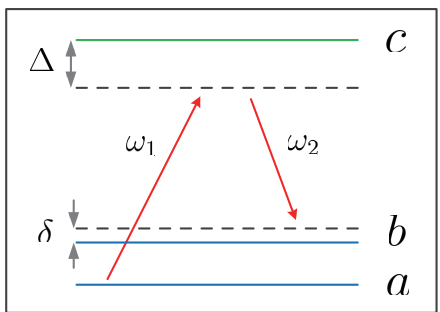

图2 (网络版彩图)拉曼光频率与原子能级示意图

Figure 2 (Color online) The $\Lambda$-type structure of Raman transition. 
差为 0 . 在考虑重力梯度时, 重力梯度会引起原子干涉 路径的不闭合. 如图3所示, 假定原子初始位置为 $z_{0}$, 初 始速度为 $v_{0}$, 对于路径I有

$z_{1}=A(T) z_{0}+B(T) v_{0}+\xi(T)$,

$v_{1}=C(T) z_{0}+D(t) v_{0}+\dot{\xi}(T)+\hbar k / M$.

对于路径II有

$z^{\prime}{ }_{1}=A(T) z_{0}+B(T)\left(v_{0}+\hbar k / M\right)+\xi(T)$,

$v^{\prime}{ }_{1}=C(T) z_{0}+D(t)\left(v_{0}+\hbar k / M\right)+\dot{\xi}(T)-\hbar k / M$.

第三束脉冲与路径I上原子作用的位置为

$z_{2}=A\left(T^{\prime}\right) z_{1}+B\left(T^{\prime}\right) v_{1}+\xi\left(T^{\prime}\right)$.

与路径II上的原子作用的位置为

$z^{\prime}{ }_{2}=A\left(T^{\prime}\right) z_{1}{ }_{1}+B\left(T^{\prime}\right) v^{\prime}{ }_{1}+\xi\left(T^{\prime}\right)$.

两条路径原子各自的速度为

$$
\begin{aligned}
v_{2}= & C\left(T^{\prime}\right) z_{1}+D\left(T^{\prime}\right) v_{1}+\dot{\xi}\left(T^{\prime}\right)-\frac{\hbar k}{M} \\
= & \left.C\left(T+T^{\prime}\right)\left(z_{0}-\frac{g}{\gamma}\right)+D\left(T+T^{\prime}\right) v_{0}+\left[D\left(T^{\prime}\right)-1\right)\right] \frac{\hbar k}{M}, \\
v_{2}^{\prime}= & C\left(T^{\prime}\right) z^{\prime}{ }_{1}+D\left(T^{\prime}\right) v^{\prime}{ }_{1}+\dot{\xi}\left(T^{\prime}\right) \\
= & C\left(T+T^{\prime}\right)\left(z_{0}-\frac{g}{\gamma}\right)+D\left(T+T^{\prime}\right) v_{0} \\
& \left.+\left[D\left(T+T^{\prime}\right)-D\left(T^{\prime}\right)\right)\right] \frac{\hbar k}{M} .
\end{aligned}
$$

这两条路径存在微小的速度差异

$v_{2}-v_{2}^{\prime}=\frac{\hbar k}{M}\left[2 \cosh \left(\sqrt{\gamma} T^{\prime}\right)-\cosh \left(\sqrt{\gamma}\left(T+T^{\prime}\right)-1\right]\right.$.

两个波包的中心位置也存在差异, 其大小为

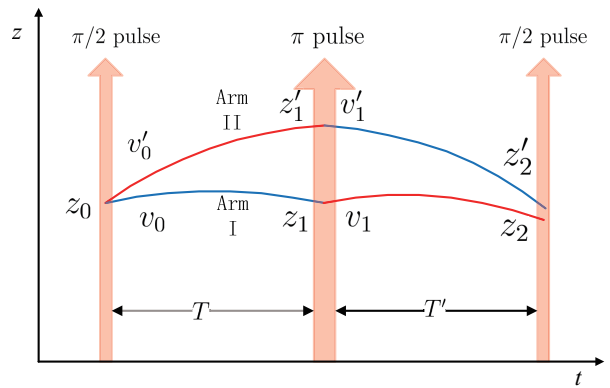

图3 (网络版彩图)原子干涉仪的空时图

Figure 3 (Color online) Space-time configuration of an atom gravimeter. $z_{2}-z^{\prime}{ }_{2}=\frac{\hbar k}{M \sqrt{\gamma}}\left[2 \sinh \left(\sqrt{\gamma} T^{\prime}\right)-\sinh \left(\sqrt{\gamma}\left(T+T^{\prime}\right)\right)\right]$

由此得到重力梯度引起的原子自由演化相位

$$
\begin{aligned}
\Delta \phi_{\mathrm{Ev}}= & \frac{1}{\hbar} \Delta S_{\mathrm{cl}} \\
= & \frac{\hbar k}{\sqrt{\gamma}}\left[2 \sinh \left(\sqrt{\gamma} T^{\prime}\right)-\sinh \left(\sqrt{\gamma}\left(T+T^{\prime}\right)\right)\right] \\
& \times\left(v_{0}+\frac{k}{2 M}\right) \cosh \left(\sqrt{\gamma}\left(T+T^{\prime}\right)\right) \\
& \left.+\sqrt{\gamma}\left(z_{0}-\frac{g}{\gamma}\right) \sinh \left(\sqrt{\gamma}\left(T+T^{\prime}\right)\right)\right] .
\end{aligned}
$$

除了原子自由演化的相位外, 激光与原子相互作 用也会引入相位. 由激光与原子相互作用引入的相位 为

$\Delta \phi_{\mathrm{LA}}=\phi_{\mathrm{eff}, z_{1}}+\phi_{\mathrm{eff}, z_{1},}-\phi_{\mathrm{eff}, \bar{z}_{2}}-\phi_{\mathrm{eff}, z_{0}}$,

其中 $\bar{z}_{2}=\left(z_{1}+z_{2^{\prime}}\right) / 2$, 在 $T=T^{\prime}$ 时, 对应的相位大小为

$\Delta \phi_{\mathrm{LA}}= \pm k_{\mathrm{eff}} g T^{2}-\alpha T^{2}$.

它包含重力引起的相移 $\Delta \phi_{g}= \pm k_{\mathrm{eff}} g T^{2}$ 以及激光扫频引 起的相移 $\Delta \phi_{\alpha}=-\alpha T^{2}$ 两个部分. 此处的正负号取决于 拉曼光波矢方向与重力加速度方向相同 $(+)$ 或者相反 $(-)$.

原子干涉仪的最终相位

$\Delta \phi=\Delta \phi_{\mathrm{Ev}}+\Delta \phi_{\mathrm{LA}}$.

包括原子自由演化的相位和激光与原子相互作用的 相位两个部分.

原子在干涉完成之后发生跃迁的概率为

$P=\frac{1}{2}(1-\cos \Delta \phi)$.

在实验中可以通过测量跃迁概率来得到原子干涉仪 的相移大小, 从而计算出 $g$ 值.

\section{2 原子干涉重力仪的技术实现}

\subsection{1 原子干涉仪工作流程}

在进行重力测量时, 首先利用红失谐的激光来对 原子团进行冷却囚禁. 红失谐激光的光子能量 $h v$ 要比 原子基态到激发态的能级差略低, 原子在受激跃迁时 会损失一部分动能. 处于激发态的原子能够通过自发 
辐射回到基态. 由于自发辐射时原子辐射光子方向是 任意的, 原子在基态与激发态之间的循环跃迁使得原 子团的温度不断降低直到多普勒冷却极限 ${ }^{[53]}$. 原子团 被冷却风禁之后可以改变上下方向激光的频差来实 现原子团的上抛. 多普勒冷却温度受限于激发态原子 自发辐射光子的反冲动量. 为进一步降低原子团的温 度, 减小自由扩散造成的原子数目损失, 在原子团上 抛时需要降低光强并且增大囚禁光整体的失谐量实 现亚多普勒冷却过程. 此过程可将原子团上抛时的温 度冷却到 $\mu \mathrm{K}$ 量级, 从而实现“运动黏团”过程 ${ }^{[44]}$.

在拉曼型原子干涉重力仪中, 原子团被冷却囚禁 上抛之后, 一束 $\pi$ 脉冲拉曼光被用于原子能态及动量态 的选择, 将原子制备到磁不敏感态上. 在清除不共振 的背景原子之后, 利用 $\pi / 2$ 脉冲将原子波包分成两束, 之后利用 $\pi$ 脉冲实现物质波的反射, 最后一束 $\pi / 2$ 脉冲 使原子的物质波汇聚以完成干涉过程. 干涉之后的原 子落到探测区时探测光可对原子的末态进行探测.

在实验中要对激光进行扫频以抵消重力引起的 多普勒频移. 改变扫频斜率的大小可以改变原子干涉 仪的相位. 通过相位的改变量以及原子末态概率之间 的关系, 可以拟合得到初始相位从而给出 $g$ 值的大小.

\subsection{2 原子干涉重力仪的真空系统}

拉曼型原子干涉重力仪有两种形式: 喷泉式原子 干涉重力仪以及自由下落式原子干涉重力仪. 美国斯 坦福小组的原子干涉重力仪便是典型的喷泉式重力 仪. 此种重力仪将原子团冷却囚禁之后上抛形成喷泉, 在原子团上抛以及下落并处于干涉区内部时进行干 涉, 这样能够在有限空间内保证较大的脉冲间隔时间 $T$. 喷泉式原子干涉重力仪还对一些系统误差具有一 定抑制作用, 如光速有限效应以及二级塞曼效应. 除 了斯坦福大学, 还有意大利佛罗伦萨大学、德国洪堡 大学、武汉物理与数学研究所以及华中科技大学等 小组采用喷泉式原子干涉重力仪. 华中科技大学的喷 泉式原子干涉重力仪结构如图4所示.

自由下落式原子干涉重力仪相比于喷泉式原子 干涉重力仪在结构上更为小巧. 由于原子团不需要上 抛, 三维磁光阱(3D-MOT)所需的六束冷却囚禁光都可 由同一个声光晶体(AOM)生成, 这也简化了光路系统. 使用自由下落式的原子干涉重力仪的小组主要有法 国巴黎天文台和浙江大学小组.

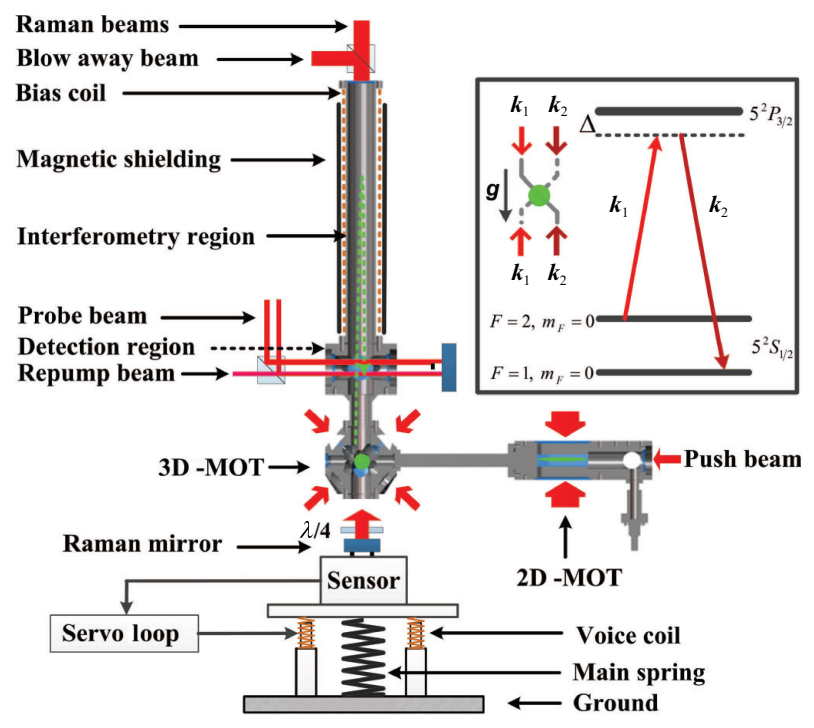

图4 (网络版彩图)华中科技大学原子干涉重力仪结构 简图 ${ }^{[8]}$

Figure 4 (Color online) The experimental setup of the atom gravimeter in Huazhong University of Science and Technology (HUST) [8].

不管是采用喷泉式还是自由下落式, 原子干涉重 力仪的真空容器对真空度都有很高要求, 一般要求达 到 $10^{-7} \mathrm{~Pa}$ 水平. 其目的是为了限制背景原子对冷原子 团的加热效应, 降低背景原子对探测性噪比的影响, 以及将拉曼光的波矢量大小 $k_{\mathrm{eff}}$ 引起的系统误差限制 在允许的范围内. 现在大部分原子干涉重力仪的结构 中除了直接囚禁原子的三维磁光阱外, 还包含对原子 进行预冷却的二维磁光阱 ${ }^{[5]}$. 二维磁光阱的目的是在 保证真空度的情况下提高原子的装载速率, 这使得原 子数目不再是重力仪分辨率的主要限制.

\subsection{3 光路系统}

原子干涉仪的光路系统用于产生原子在冷却囚 禁、干涉及探测时所需的各个频率的激光. 原子干 涉仪通常采用碱金属原子进行干涉. 在光路中, 一台 DBR激光器锁在固定频率用作回百光以及拉曼光的 参考光, 两台窄线宽的外腔式半导体激光器拍频锁相 后形成拉曼光, 另外还需一台激光器来提供冷却囚禁 光. 也有小组利用两台外腔半导体激光器拍频锁相形 成冷却囚禁光及回百光. 然后利用声光晶体将混叠后 的主从激光移频 $1 \mathrm{GHz}$ 左右, 产生所需拉曼光, 其中本 课题组的光路便采用了这种形式, 如图 5所示. 在实验 室中的光路大多采用窄线宽的半导体激光器来生成低 


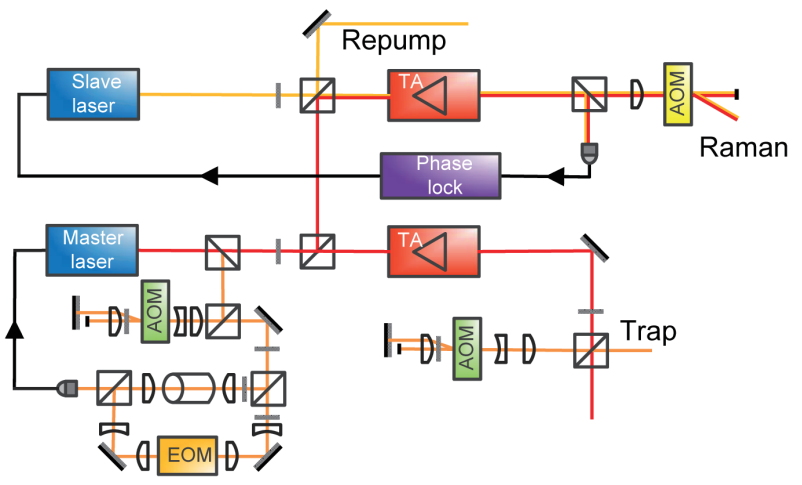

图5 (网络版彩图)华中科技大学原子干涉仪的光路配置 Figure 5 (Color online) Optical scheme of the atom interferometer in HUST.

相位噪声的拉曼光. 半导体激光器对环境的温度、振 动等因素要求较高, 这不利于重力仪在环境因素变化 较大的场所内的正常工作.

随着原子干涉重力仪技术的发展, 近年来也有 小组利用商用的 $1560 \mathrm{~nm}$ 光纤激光器或者DFB激光器 采用倍频晶体倍频的方法来得到所需的 $780 \mathrm{~nm}$ 的激 光. 相比于外腔半导体激光器, 它们的稳定性更好, 有 利于原子干涉仪的商业化应用, 本文将在第2.3节原子 干涉重力仪的小型化2.3.2部分中着重介绍此方面的 发展.

\section{3 原子干涉重力仪的小型化}

\subsection{1 真空系统的小型化}

原子干涉仪从实验室中的固定测量仪器向可移 动化方向发展的过程中, 最先面临的装置的小型化问 题. 实验室中的真空容器通常有较长的干涉区用于 增大原子干涉仪的面积, 提高信号的幅度, 这也使得真 空容器的体积庞大, 难以搬运. 在可移动原子干涉重 力仪的研制过程中, 各个小组也在保证测量分辨率的 前提下尽量减小真空容器体积大小 ${ }^{[23]}$. 现阶段分辨率 达到 $10 \mu \mathrm{Gal} / \sqrt{\mathrm{Hz}}$ 的可移动原子干涉重力仪高度都已 限制在 $2 \mathrm{~m}$ 以内, 能够搬运至不同地点用于重力测量.

除了限制干涉区的长度外, 有的小组正在进行真 空容器结构简化的研究. 法国巴黎天文台进行了单 束光实现原子冷却囚禁、干涉及探测的实验 ${ }^{[56]}$, 在 2010 年分辨率达到了 $170 \mu \mathrm{Gal} / \sqrt{\mathrm{Hz}}$ 的水平. 此外各个 小组在设计可移动原子干涉仪时也将光路进行了小
型化 ${ }^{[57]}$

\subsection{2 光路的小型化}

在可移动原子干涉重力仪中, 由于环境的噪声通 常比实验室中更为明显, 实验环境的变化也对光路系 统的稳定性有更高的要求. 通常在实验室中使用的外 腔式半导体激光器线宽较窄, 也对环境的要求更为苛 刻. 这不利于原子干涉重力仪在外部温度及振动变化 明显的环境中长期稳定测量.

在各个原子干涉仪的研究小组中, 也有将通讯波 段DFB激光器倍频技术用于原子干涉重力仪的, 其光 路的配置简图如图6所示. 倍频晶体(PPLN)能够将DFB 激光器发出的 $1560 \mathrm{~nm}$ 的激光器倍频, 从而产生 $780 \mathrm{~nm}$ 的激光; 电光调制器(EOM)用于调制激光产生 $6.8 \mathrm{GHz}$ 边带, 生成回原光及拉曼光; 掺铒光纤放大器 $(\mathrm{EDFA})$ 用于激光功率的放大.

法国航天实验室小组将铒光纤DFB激光器用于 原子干涉仪的光路 ${ }^{[58]}$, 其线宽达到了 $2.5 \mathrm{kHz}$. 他们的 光路系统更为简洁, 只用了一台激光器. 激光器出来 的光经过激光相位调制器后移频 $1 \mathrm{GHz}$ 的一级边带被 直接锁在 ${ }^{85} \mathrm{Rb}$ 的 $\mathrm{D} 2$ 跃迁线. 在实验中, 通过调节激光 相位调节器的驱动频率来使零级边带频率对应 ${ }^{87} \mathrm{Rb}$ 的 $F=2$ 到 $F^{\prime}=3$ 跃迁线, 从而生成冷却囚禁光. 在生成拉曼 光时, 只需改变 $1 \mathrm{GHz}$ 激光相位调节器的驱动频率, 并 且利用另外一个激光相位调节器移频 $6.8 \mathrm{GHz}$ 产生边 带生成拉曼光.

浙江大学小组利用 $1560 \mathrm{~nm}$ 的DFB激光通过倍频 晶体倍频得到 $780 \mathrm{~nm}$ 的激光 ${ }^{[59]}$. 在实验中, 浙江大学 小组利用一台激光器锁在 ${ }^{85} \mathrm{Rb}$ 的 $\mathrm{D} 2$ 跃迁线上作为参 考光, 另一台激光器与参考激光器拍频通过数字跳频

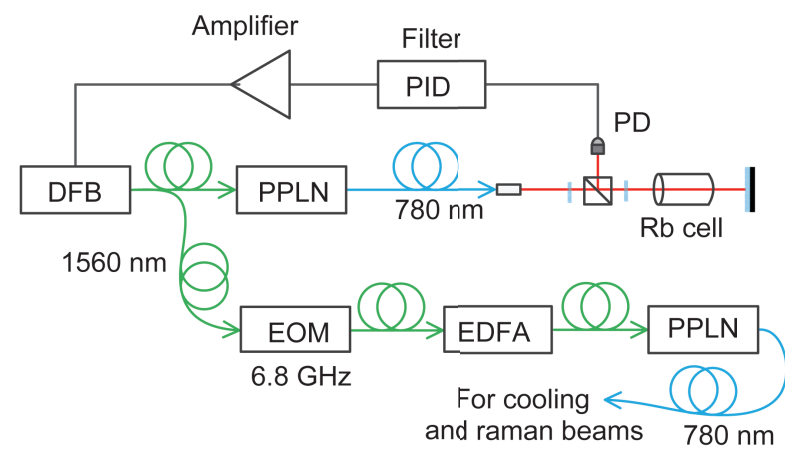

图6 (网络版彩图) 光纤倍频光路示意图

Figure 6 (Color online) Diagram of the frequency-doubled fiber laser 
锁定技术锁定在 ${ }^{87} \mathrm{Rb}$ 的 $F=2$ 到 $F^{\prime}=3$ 跃迁线上形成冷却 贝禁光, 回泵光由电光相位调制器移频 $6.8 \mathrm{GHz}$ 后生 成. 实验中相位相干的拉曼光由主激光以及电光相位 调制器调制后的光生成.

采用DFB或DBR激光器以及激光相位调制器产 生相位相干光的方法, 比外腔半导体激光器及光学锁 相环产生相干光的方法稳定性更高. 本课题组也在进 行此方面的技术研究, 有望为其可移动重力仪提供稳 定的光路系统.

\section{4 原子干涉重力测量的噪声}

为实现高精度的重力测量, 在实验中首先需要抑 制测量过程中的噪声以提高测量的分辨率. 目前, 原 子干涉重力测量的主要噪声包括地面振动噪声、拉 曼光相位噪声、探测噪声等. 对于各项主要噪声的抑 制各小组也有不同的方法, 此节将简要介绍这些噪声 的抑制方法.

\subsection{1 振动噪声}

原子干涉重力仪测量的是原子团相对于拉曼反 射镜的加速度, 因此拉曼反射镜的惯性加速度会影响 原子的重力加速度的测量. 在整个干涉过程中, 拉曼 反射镜的振动会影响激光与原子相互作用的相位大 小, 从而引起原子干涉仪的相位变化. 在实验中对这 部分噪声进行抑制的方法通常有直接测量振动噪声

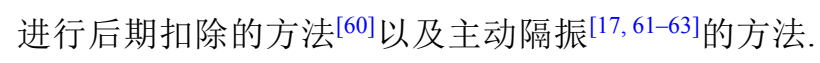
本课题组采用了主动隔振的方法来对振动噪声进行 抑制[21].

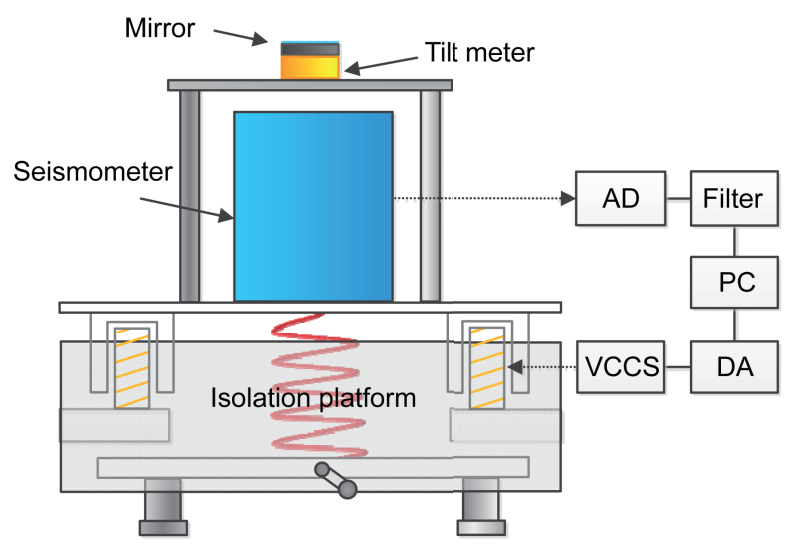

图7 (网络版彩图)主动隔振装置原理图

Figure 7 (Color online) Configuration of the active vibration isolator.
主动隔振装置由被动隔振平台、微震仪、力学 反馈以及滤波装置组成, 如图7所示. 在实验中, 微震 仪测量到的平台残余振动信号被滤波之后用于平台 的力学反馈. 利用此项技术, 1-10 Hz频段的残余振动 噪声能够被抑制 1 个量级左右, 从而降低残余振动对 重力测量噪声的贡献. 斯坦福大学、德国洪堡大学、 武汉物理与数学研究所以及华中科技大学的原子干 涉重力仪采用这种方法来抑制振动噪声.

法国巴黎天文台 ${ }^{[18]}$ 小组发展了另一套技术用于 振动噪声的抑制, 如图8所示. 他们利用微震仪直接测 量平台的振动, 然后进行滤波之后直接用于重力测量 数据的相位后期修正, 从而达到了抑制振动噪声的效 果. 国内浙江大学也采用这种数据后处理的方式.

\subsection{2 拉曼光相位噪声}

原子干涉重力仪通过测量重力引起的干涉相移 的方法来确定重力大小, 拉曼光的相位噪声会影响最 终的重力测量分辨率, 因此实验中的一个重要的技术 环节是相位相干的拉曼光的获得. 实验中获得两束固 定频差且相位相干的激光的手段主要有声光晶体移

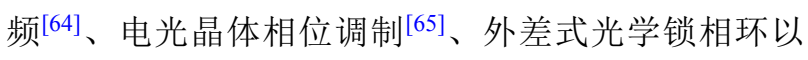
及外差光注入锁定结合锁相环的方法 ${ }^{[66,67]}$. 目前本课 题组实验中选取光学锁相的方法实现两台激光器的 相位锁定.

在光学锁相环中, 华中科技大学利用拉曼光拍频 信号与另外一台相噪水平更好的微波源(SDI频率合 成器)进行混频得到低频信号, 此低频信号与参考DDS 信号源鉴频鉴相之后用于激光器的反馈控制. 拍频锁 相环的噪声如图9所示. 光学锁相环在频率 $10 \mathrm{kHz}$ 处

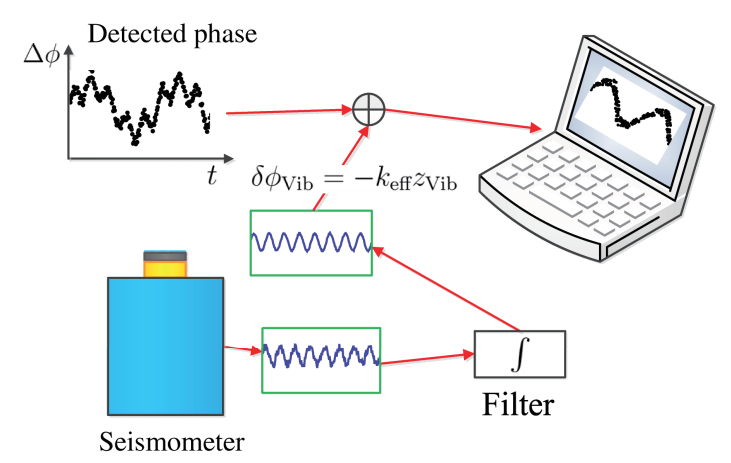

图8 (网络版彩图)原子干涉仪相位的振动噪声修正 Figure 8 (Color online) Phase correction of vibration signal in atom interferometer. 


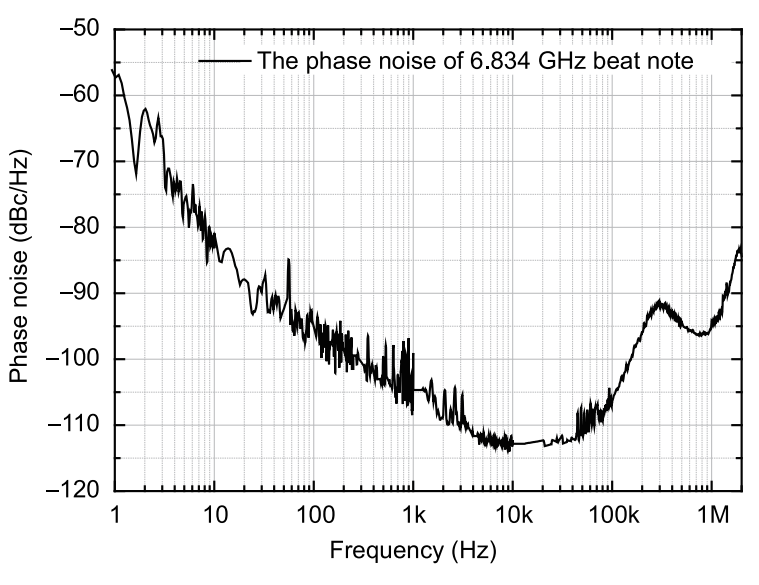

图9 (网络版彩图)光学锁相环相位噪声谱

Figure 9 (Color online) Phase noise spectrum of the optical phase lock loop.

的相位噪声达到了近 $-110 \mathrm{dBc} / \mathrm{Hz}$.

在实验上产生相位相干拉曼光的另一种方法是电 光相位调制法. 此种方法多用于光纤激光器以及DFB 激光器生成相位相干的拉曼光. 利用该方法相位噪声 对单次重力测量的影响可以抑制到 $2.6 \mu \mathrm{Gal}$ 的水平 ${ }^{[58]}$. 此外利用电光调制配合法布里-珀罗腔进行滤波的方 法也被用于DFB激光器产生低相噪的相干拉曼光的 实验中 ${ }^{[68]}$.

\section{5 原子干涉重力测量分辨率}

原子干涉重力测量的长期记录结果给出了重力 值变化的潮汐数据, 如图10所示. 扣除重力潮汐之后 的残差可以计算得出艾伦方差的大小, 从而得到原子 干涉重力仪的短期测量分辨率.

现阶段原子干涉重力仪的物质波操控方法主要有 受激拉曼跃迁、布拉格衍射以及光晶格操控几种方 法. 其中分辨率做得最好的是拉曼型原子干涉重力仪.

在拉曼型原子干涉仪中, 法国的航天实验室 小组用于场效应测量的可移动原子干涉仪 ${ }^{[69]}$ 达到 了 $42 \mu \mathrm{Gal} / \sqrt{\mathrm{Hz}}$, 武汉物理与数学研究所的喷泉式 原子干涉重力仪达到了 $110 \mu \mathrm{Gal} / \sqrt{\mathrm{Hz}}$ 的水平, 浙 江大学小组用于场效应测量的原子干涉仪 ${ }^{[20]}$ 达到 了 $100 \mu \mathrm{Gal} / \sqrt{\mathrm{Hz}}$, 德国洪堡大学的可移动原子干涉重 力仪 ${ }^{[70]}$ 达到了 $9.6 \mu \mathrm{Gal} / \sqrt{\mathrm{Hz}}$, 法国巴黎天文台的可移 动重力仪 ${ }^{[71]}$ 分辨率达到了 $5.7 \mu \mathrm{Gal} / \sqrt{\mathrm{Hz}}$, 斯坦福大学 的重力仪 ${ }^{[2]}$ 分辨率达到了 $8 \mu \mathrm{Gal} / \sqrt{\mathrm{Hz}}$, 本课题组的重 力仪 ${ }^{[8]}$ 分辨率为 $4.2 \mu \mathrm{Gal} / \sqrt{\mathrm{Hz}}$.
除了拉曼型原子干涉仪外, 澳大利亚小组利用 原子的布拉格衍射操控原子动量态进行干涉的方法 测量重力加速度 ${ }^{[72]}$, 积分 $1000 \mathrm{~s}$ 之后测量分辨到了 2.7 $\mu \mathrm{Gal}$. 意大利小组研制的的光晶格原子干涉重力仪不 确定度也达到了 $10^{-7} \mathrm{~g}$ 水平. 各小组重力仪的分辨率 如表1所示.

\section{6 原子干涉重力测量系统误差评估}

原子干涉重力仪是绝对重力仪, 这要求重力测量 在保证高分辨率的情况下要对原子干涉仪的系统误 差进行评估. 原子干涉重力测量的系统误差可分为两 类, 第一类和拉曼光的波矢 $k_{\mathrm{eff}}$ 方向没有明显关系, 在 重力测量中, 可以通过改变拉曼光波矢方向的方法对 此类系统误差进行抑制 ${ }^{[34]}$. 但在激光反转波矢方向 时, 由于原子吸收光子后反冲的方向不同会导致 $\pm k_{\mathrm{eff}}$ 配置下原子的路径有所不同, 反转波矢方向的方法不 能完全抑制与波矢方向无关的系统误差. 另一类是与 波矢方向相关的系统误差, 此类系统误差通常是重力
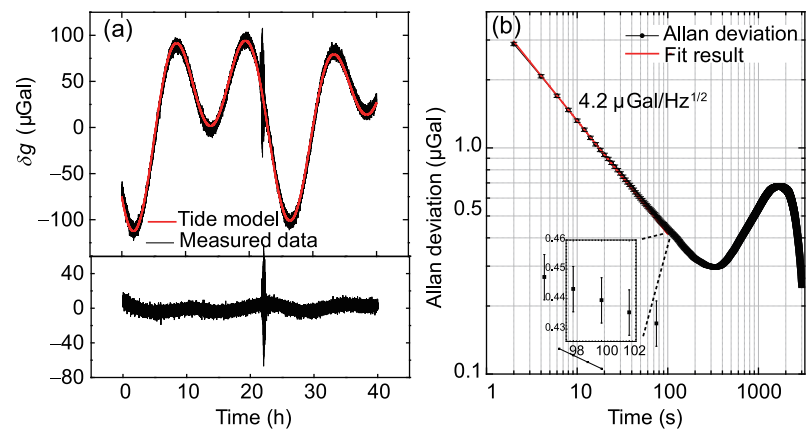

图10 (网络版彩图)重力测量数据(a)及其艾伦方差(b)

Figure 10 (Color online) (a) Results of measuring g, (b) the Allan deviation of the results.

表1 国内外原子干涉重力仪小组的分辨率

Table 1 Sensitivities of atom interferometry gravimeters in China and the other countries

\begin{tabular}{|c|c|}
\hline 原子干涉重力仪小组 & 分辨率 $\left(\mu \mathrm{Gal} / \mathrm{Hz}^{1 / 2}\right)$ \\
\hline 法国航天实验室[69] & 42 \\
\hline 法国巴黎天文台 ${ }^{[71]}$ & 5.7 \\
\hline 武汉物理与数学研究所 ${ }^{[19]}$ & 110 \\
\hline 浙江大学 [59] & 100 \\
\hline 德国洪堡大学 [70] & 9.6 \\
\hline 斯坦福大学[2] & 8 \\
\hline 华中科技大学[8] & 4.2 \\
\hline 澳大利亚布拉格型 ${ }^{772]}$ & 85 \\
\hline
\end{tabular}


测量中的主要系统误差项. 在实验中需要单独调节某 个物理量来评估相应的效应影响大小.

在原子干涉仪中, 与波矢方向无关的系统误差主 要有塞曼效应与二级斯塔克效应, 与波矢相关的效应 较多, 例如拉曼光的倾斜、重力梯度效应、萨奈克效 应等. 本文在此主要介绍一些在原子干涉重力测量中 较为明显的效应。

\subsection{1 塞曼效应}

在重力测量实验中, 为抑制磁场梯度对重力测量 的影响, 原子被制备在 $m_{F}=0$ 态, 这样能够很好地抑制 一级塞曼效应, 但 $m_{F}=0$ 态原子的二级塞曼效应的影响 仍有待评估. 在实验中可以利用拉曼谱的方法测量出 干涉区的磁场大小从而得到原子演化时间内的磁场变 化, 并以此计算出磁场对重力测量的系统误差的贡献.

由于原子干涉重力测量对磁场的梯度敏感, 本课 题组提出了将原子制备在磁敏感态测量磁场梯度来 评估塞曼效应的方法 ${ }^{[42]}$. 在此项系统误差评估中, 通 过将原子制备在 $m_{F}= \pm 1$ 的磁敏感态的方法来对磁屏蔽 之后的干涉区的磁场梯度进行测量, 计算出磁场梯度 对重力测量结果的系统误差贡献. 通过精确的测量磁 场梯度, 塞曼效应对重力测量的影响可以评估到 0.2 $\mu \mathrm{Gal}$ 的水平.

\subsection{2 二级斯塔克效应}

在原子干涉仪中, 拉曼光的交变电场会引起原子 两个基态能级移动, 从而改变它们的能级差, 能极差 的大小与拉曼光的两个频率成分的光强大小相关. 在 实验中, 可以通过调节拉曼光的两个成分的光强比来 调节光频移的大小.

朱棣文小组 ${ }^{[15]}$ 提出了一种测量二级斯塔克效应 的方法, 如图11所示, 通过在前一个 $T$ 时间内或者第二 个 $T$ 时间内额外增加一个非共振的拉曼脉冲, 可以得 到激光脉冲的光频移对干涉仪相位的影响大小. 通过 改变脉冲的作用时间对这部分相位进行大小调制. 脉 冲的时间越长, 对相位的影响越大. 在实验中, 额外的 激光脉冲加在 $\pi$ 脉冲之前与之后引起的相位变化方向 相反. 额外光脉冲引起相位的变化除了与作用时间相 关外, 还与拉曼光的光强比例相关, 当光强比例调节 合适时, 光频移的绝对大小可以降至最小. 朱棣文小 组通过此种方法降低光频移的绝对大小, 使得光频移

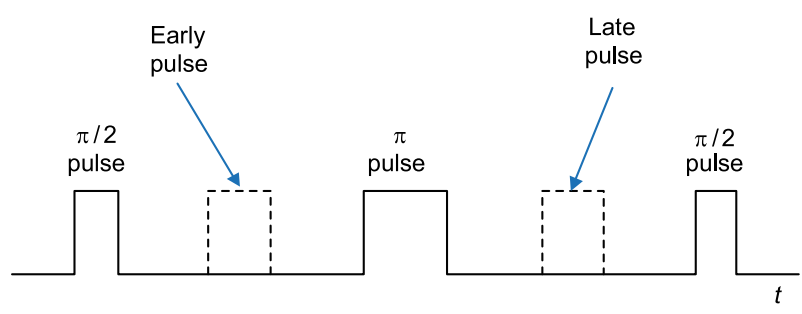

图11 (网络版彩图)二级斯塔克频移的评估实验脉冲时序 图

Figure 11 (Color online) Configuration of Raman pulses in the evaluation experiment of AC Stark shift.

效应造成的影响小于 $1 \mu \mathrm{Gal}$ 水平.

本课题组则利用微波使原子跃迁来测量光频移 的大小. 原子被激光照射时存在光频移, 这能改变能 级差的大小, 从而引起微波共振跃迁频率的变化. 通 过没有激光时微波的共振跃迁频率 $f_{0}$, 以及非共振拉 曼光照射下微波的跃迁频率 $f_{1}$ 之间的变化 $\Delta f=f_{1}-f_{0}$, 得到了光频移的相对大小. 通过适当调节拉曼光两个 频率成分的功率比, 将拉曼光功率比引起的光频移影 响抑制到 $0.1 \mu \mathrm{Gal}$ 的水平.

此外,由于原子在重力场中自由下落, 在此过程 中需要对拉曼光进行扫频来抵消重力引起的失谐量 变化. 由于激光扫频作用导致三束脉冲时光强 ${ }^{[73]}$ 及失 谐量 $\Delta$ 的变化也将引起二级斯塔克频移. 巴黎天文台 小组对失谐量 $\Delta$ 的影响进行了讨论, 由此引起的系统 误差在 $\mu \mathrm{Gal}$ 量级 ${ }^{[74]}$.

\subsection{3 拉曼光方向倾斜}

原子干涉仪进行重力测量时, 重力加速度引起的 相位由重力加速度以及拉曼波矢共同决定

$\phi_{\theta}=\boldsymbol{k}_{\text {eff }} \boldsymbol{g} T^{2}=\left|\boldsymbol{k}_{\text {eff }}\right| \boldsymbol{g} \mid T^{2} \cos \theta_{i}$.

斯坦福大学小组在评估倾斜引起系统误差时, 利 用倾斜仪来测量拉曼反射镜的角度变化. 通过沿某个 特定方向旋转反射镜, 得到了倾斜方向改变与重力测 量值之间的关系曲线. 重力值处于极大值时, 可以找到 坚直方向对应的倾斜度数. 倾斜仪的精度为 $0.1 \mu \mathrm{rad}$, 由此方法测量的倾斜角度绝对精度在 $5 \mu \mathrm{rad}$ 以内.

在测量出拉曼反射镜的倾斜方向之后, 斯坦福小 组利用倾斜仪的读数对光学桌的倾斜角度进行了反 馈控制. 在进行倾斜控制之后, 光学桌短期稳定度能 达到 $1 \mu \mathrm{rad}$, 长期稳定度好于 $20 \mu \mathrm{rad}$, 最终倾斜的影响 
对 $g$ 值的系统误差的贡献在 $0.1 \mu \mathrm{Gal}$ 以内.

本课题组也做了相关调制实验, 实验配置如图12所 示. 通过在隔振平台上添加质量块的方法改变平台的 倾斜, 在平台的倾斜改变之后, 还需调节拉曼光扩束 器的角度来使得扩束器出射光的方向和反射光的方 向一致. 调制实验的结果如图13所示, 由于倾斜存在 两个方向的变化, 需要首先进行一个方向的调制实验, 将该方向倾斜调到中心值. 一个方向倾斜调节到中心 值之后, 再进行另外一个方向上的倾斜调制实验. 通 过调制实验, 将倾斜引起的系统误差评估到了 $0.1 \mu \mathrm{Gal}$ 水平.

\subsection{4 重力梯度}

重力是地球表面物体受到的地球的万有引力以 及惯性离心力的合力. 万有引力场的大小随着测量点 距引力场的中心距离增大而衰减, 在重力中体现为地 球表面物体随高度增加受到的重力减小, 即坚直重力 梯度效应. 原子干涉重力仪测量的是整个测量过程中 原子的重力加速度的平均值. 不可避免的, 重力梯度 将对测量结果有所贡献.

由公式(19)可以得到重力梯度的一阶影响大小为

$\Delta g_{\gamma}=\gamma\left(\frac{7}{12} g_{0} T^{2}-v_{0} T-z_{0}\right)$,

其中 $g_{0}, v_{0}, z_{0}$ 分别是第一个拉曼脉冲时刻的重力加速

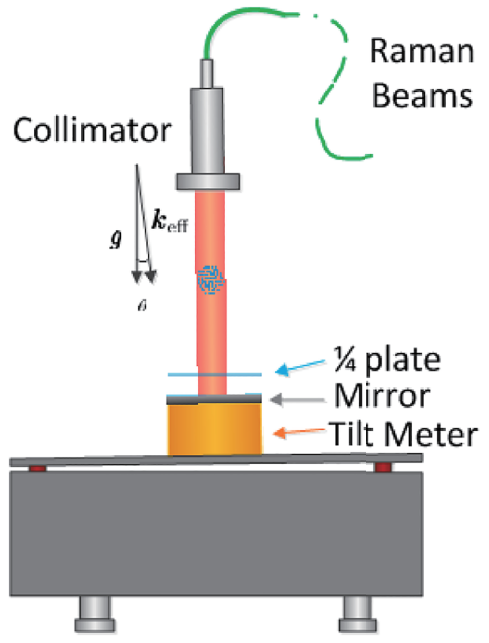

图12 (网络版彩图) 倾斜调制实验的实验设置

Figure 12 (Color online) Experimental configuration of the tilt modulation experiments.
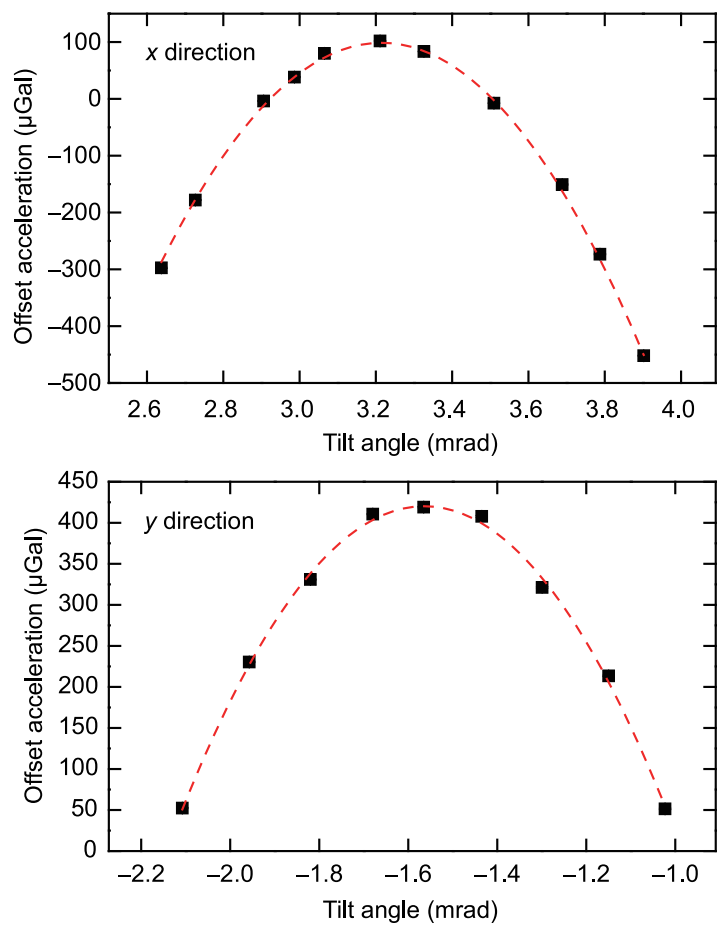

图13 (网络版彩图)倾斜调制实验结果

Figure 13 (Color online) The results of tilt modulation experiment.

度、速度以及位置. 在实验中可以利用原子干涉仪 测量重力的线性梯度从理论上扣除重力梯度的影响. $T=200 \mathrm{~ms}$ 时, 将重力梯度测量到 $30 \mathrm{E}\left(1 \mathrm{E}=10^{-9} \mathrm{~s}^{-2}\right)$, 可 以把重力梯度引起的系统误差评估到 $1 \mu \mathrm{Gal}$ 水平.

\subsection{5 科里奥利力}

地球自转会使得拉曼光方向也会随着一起转动, 假如初始速度有一个水平分量的话, 它在三束脉冲的 方向上投影大小不同, 等效存在一个重力加速度的变 化. 由公式 ${ }^{[15]}$

$\Delta g_{\Omega_{\oplus}}=\left(\boldsymbol{\Omega}_{\oplus} \times \boldsymbol{e}_{k_{\text {eff }}}\right) \cdot \overline{\boldsymbol{v}}$

地球的转动速度 $\Omega_{\oplus}=7.292 \times 10^{-5} \mathrm{rad} / \mathrm{s}$, 通常原子 干涉重力测量中的科里奥利力效应可以达到 $10 \mu \mathrm{Gal}$ 水平.

斯坦福大学朱棣文小组采用改变探测区东西方 向位置并引入转动调制的方法来寻找科里奥利力效 应最小的点. 通过引入额外转动, 只有在科里奥利力 效应最小的点对应的 $g$ 值变化最小.

加州大学伯克利小组则利用Ramsy-Bordé型干涉 
仪直接测量科里奥利力引起的相位变化来评估它引起 的效应大小 ${ }^{[75]}$, 之后利用旋转拉曼反射镜方向来抵消 地球自转的方法来抑制科里奥利力引起的系统误差.

\subsection{6 波前畸变}

目前, 原子干涉重力测量准确度的主要限制因素 是拉曼光的波前畸变. 理论上, 原子干涉仪中的拉曼 光被视作平面波, 但是实际的拉曼光并不是理想的平 面波, 这会导致一个系统误差, 这在原子干涉重力测量 中被称作波前畸变. 如图14所示, 波前畸变不仅与拉 曼光波前的畸变相关, 还与原子团的分布相关. 实验 上来说, 主要由三个方面的原因导致拉曼光不是平面 波: 拉曼激光本身是高斯光束, 波前本身是非平面的; 光学元件表面的灰尘等散射体; 光学元件的不完美, 导 致反射回来的拉曼光产生畸变. 本课题组的拉曼光直 径约 $26 \mathrm{~mm}$, 光斑大小的影响较小. 现阶段原子干涉重 力测量中波前畸变主要来自于不完美的光学元器件.

1994年, 斯坦福大学朱棣文小组 ${ }^{[44]}$ 在用原子干涉 仪测量精细结构常数时就认识到并理论计算了波前 弯曲的影响以及光学元件表面散射体对拉曼光波前 相位的影响. 他们在后来的原子干涉重力测量中则通 过实验验证了灰尘等表面散射体的影响 ${ }^{[15]}$, 另外还通 过移动拉曼光扩束器中准直透镜的位置来改变拉曼 光波前曲率半径从而评估出拉曼光波前弯曲的影响.

法国巴黎天文台的实验表明, 波前畸变除了与光 学元件表面质量有关, 还与原子团分布相关. 他们的 实验通过改变原子团温度来推测原子团温度为 0 时的 $g$ 值, 进而得到通常的原子团温度下波前畸变的大小 为 $4 \mu \mathrm{Gal}^{[34]}$.

改变原子团温度是一个直接改变原子团分布的

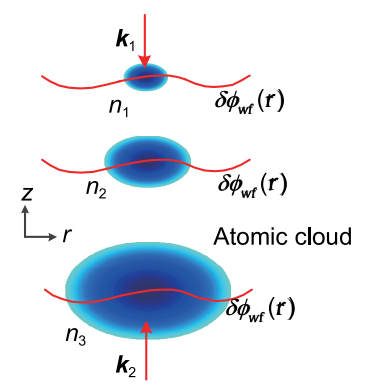

图14 (网络版彩图)原子团扩散及波前畸变示意图 Figure 14 (Color online) The expansion of atomic cloud and wave front aberration.
办法,而选择不同分布范围的原子也等效地改变了原 子团的分布. 德国柏林洪堡大学Peters小组 ${ }^{[76]}$ 的实验, 在拉曼光光路中插入已知表面分布的平板玻璃, 通过 改变探测区的半径来选择不同分布范围的原子,进而 改变波前畸变对 $g$ 值的贡献. 但波前畸变的绝对大小 仍有待确定, 因为抽真空前后容器窗口的形变仍无法 完全确定.

\subsection{7 光速有限效应}

光速有限效应主要包括两个部分, 第一部分是由 于三个脉冲时刻原子位置的变化引起的光速效应; 第 二部分是激光扫频和光速有限共同作用对重力测量 值的影响, 如图 15所示.

斯坦福大学小组对由原子位置变化引起的光速 有限效应进行了估算. 巴黎天文台小组对扫频引起的 效应进行了计算, 并且进行了实验上的验证 ${ }^{774]}$.

对于原子干涉重力仪, 共有 3 个拉曼光脉冲与原 子相互作用, 每两个脉冲之间的间隔为 $T$, 每一个光脉 冲均由两束对射的光 $\boldsymbol{k}_{1}$ 和 $\boldsymbol{k}_{2}$ 构成. 激光器发射的拉曼 光脉冲间隔为 $T$, 拉曼光从激光器发出到作用到原子 上, 这一段的时间延迟效应便称为有限光速效应, 它 会对 $g$ 的测量造成一个系统偏差. 高精度的原子干涉 重力仪中有限光速效应作为一项系统误差应该被详 细地考虑.

原子干涉重力仪中的有限光速效应首次是被朱 棣文小组 ${ }^{[15]}$ 进行研究的, 他们通过简要的分析, 得到 包含了有限光速效应的 $g$ 的表达式为

$g=g_{0}\left(1+2 \frac{v_{\pi}}{c} \frac{\alpha_{1}+\alpha_{2}}{\alpha_{1}-\alpha_{2}}\right)$

其中 $v_{\pi}$ 为 $\pi$ 脉冲时刻原子的速度, $c$ 为光速, $\alpha_{1}$ 和 $\alpha_{2}$ 分别

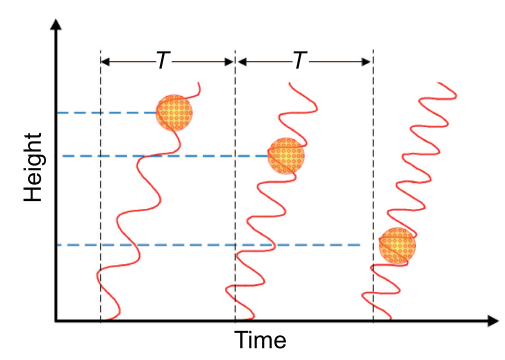

图15 (网络版彩图)原子干涉仪的光速有限效应 Figure 15 (Color online) The finite speed of light effect in atom interferometers. 
为 $\boldsymbol{k}_{1}$ 光和 $\boldsymbol{k}_{2}$ 光的扫频斜率. 表达式(27)表明, 有两种方 式几乎可以消除这种有限光速效应: (1) 使 $\pi$ 脉冲的中 心与原子团运动的顶点重合; (2) 使两个扫频斜率等 值反号, 即 $\alpha_{1}+\alpha_{2}=0$. 已经有小组从实验上证实了表达 式中所示的有限光速效应对 $g$ 值的影响 ${ }^{[74]}$.

Dimopoulos等人 ${ }^{[43,77]}$ 根据原子干涉仪的工作原理, 从广义相对论的角度计算了原子干涉重力仪的总相 移, 由于计算量较大, 他们借助了计算机完成计算过 程. 从他们所给的表达式中分离出的仅含有有限光速 效应的结果为

$g=g_{0}\left(1+3 \frac{v_{\pi}}{c}\right)$

表达式(28)给出了一个与角雉型绝对重力仪的有限光 速效应类似的结果.

对比式(27)和(28), (27)给出了一个与拉曼光的扫 频斜率耦合的有限光速效应, 式(28)给出了一个与扫 频无关的结果. 因此较为完整的有限光速效应需要进 一步探索.

\subsection{8 仪器自身引力}

由于万有引力的不可屏蔽性, 除地球外的其他物 体对原子的万有引力会影响重力测量, 在星体则体现 为潮汐, 测量人员的万有引力则体现为噪声变化, 原 子干涉仪自身的引力则会引起系统误差. 由于万有引 力大小是与距离的平方成反比的, 因此仪器自身的引 力的影响显得尤为重要.

在原子干涉仪中, 由于测量的是重力加速度在一 段时间内的平均值, 万有引力的大小又是随距离变化 的物理量, 因此需要慎重地分析各个部分的影响. 巴 黎天文台评估了由于局部质量分布引起的重力测量 的系统误差 ${ }^{[78]}$, 本课题组也对实验装置自身引力引起 的系统误差进行了评估, 评估的精度达到了 $0.1 \mu \mathrm{Gal}$ 水平.

\subsection{9 环境因素}

由于地球表面崎岖不平、变化万千, 使得重力场 随着质量分布的空间变化而改变; 日月星辰的更替变 换, 江河湖泊的枯满盈亏, 使得同一地点的重力也随 时间变化. 综合起来说, 重力场是一个随时间、空间 位置变化的物理量, 当然重力场的变化也能反映地球 的质量分布以及日月引力大小的时空变化.
为扣除背景重力的变化, 除了要建立可靠的重力 潮汐模型外, 仪器间的相互比对也是得到背景重力变 化的合理方式. 在国际上也有相应的重力比对来检验 重力测量仪器的测量精度, 分析各个仪器可能存在的 系统误差, 得到一个合理的重力测量值. 由国际计量 局(BIPM)组织的国际绝对重力仪比对(ICAG)自 1981 年来每四年进行一次. 在国际重力仪比对期间, 数十 个绝对重力仪被汇集到一起来测量局部的重力值大 小. 巴黎天文台的可移动原子干涉绝对重力仪参与了 2009年国际计量局举行的第八届ICAG以及2013年在 卢森堡举行的第九届ICAG. 原子干涉重力仪与经典的 绝对重力仪共同比对为各自系统误差的评估提供了 重要参考 ${ }^{[79]}$.

\section{3 原子干涉仪其他应用}

\section{1 差分测量重力梯度}

利用重力仪进行同时差分测量重力梯度具有能 抑制共模噪声的优点. 例如地面振动噪声和拉曼光相 位噪声. 近几年来, 基于原子干涉技术的重力梯度仪 迅速发展. 第一台原子重力梯度仪是由斯坦福大学的 Kasevich组研制的 ${ }^{[34]}$. 如图16(a)中所示, 两个相隔1.4 $\mathrm{m}$ 的磁光阱同时上抛原子形成两个原子干涉仪. 两个 原子干涉仪共用同一束拉曼光. 这个原子重力梯度仪 的短期分辨率为 $30 \mathrm{E} / \sqrt{\mathrm{Hz}}$. 如图 $16(\mathrm{~b})$ 所示为意大利佛 罗伦萨大学Tino小组的原子干涉重力仪 ${ }^{[36]}$, 它只有一 个磁光阱, 采用的是预抛原子的方案, 这个梯度仪结 构更加简单, 差分加速度分辨率达到了 $3 \mu \mathrm{Gal} / \sqrt{\mathrm{Hz}}$.

在基于受激拉曼跃迁的原子干涉仪中, 传统的运 行方式是扫描拉曼光引起的相位, 记录完整的干涉条 纹. 然而, 在同一个干涉条纹中, 处于极值点处的点对 于相位波动没有处于条纹中心处的点灵敏. 这将会降 低整体的测量分辨率. 事实上, 让原子干涉仪一直工 作在干涉条纹的中点来提高测量分辨率的方法已经 应用在原子干涉重力仪中, 这就是条纹锁定的方法 ${ }^{[80]}$. 在条纹锁定方法中, 激光引起的相位不再一步一步扫 描来获取完整条纹, 而是调制拉曼光的相位将原子干 涉仪的相位锁定在条纹中心, 并记录反馈相位. 这种 实时的反馈能够保持原子干涉仪在无论重力波动还 是其他扰动引起相位变化的情况下都一直工作在条 纹中心. 
原子重力梯度仪一般都包括两个同时运行的原 子干涉仪, 所以条纹锁定的方法也理应能够提高原子 重力梯度仪的分辨率. 然而, 仅仅通过调制拉曼光的 相位来锁定两个具有独立干涉相位的干涉仪在实验 上存在较大困难. 在这个问题上, 通过增加一路反馈 控制的方法, 本课题组将两个干涉仪同时锁定在各自 的条纹中心处, 使二者同步运行. 采用条纹锁定的方 法实现了 $670 \mathrm{E} / \sqrt{\mathrm{Hz}}$ 的重力梯度测量分辨率, 实验装 置如图 $16(\mathrm{c})^{[81]}$ 所示. 这个反馈控制是通过一个通电 线圈引入磁场梯度, 磁场梯度产生的相位参与反馈控
制. 如图17(a)所示为上下两个干涉仪条纹锁定前后的 条纹. 这种双条纹锁定的方法能够使测量结果对差分 重力信号更敏感，同时能够使测量方程线性化，因此 每两次测量就能够直接获得差分相位信号. 这种双条 纹锁定的方法仍然保留了原子干涉梯度仪共模噪声 抑制的优势, 并且能够将数据采样率提高一个数量级, 将分辨率提高3倍, 如图16(b)所示.

\section{2 万有引力常数测量}

原子干涉法为牛顿万有引力常量 $G$ 的测量提供了
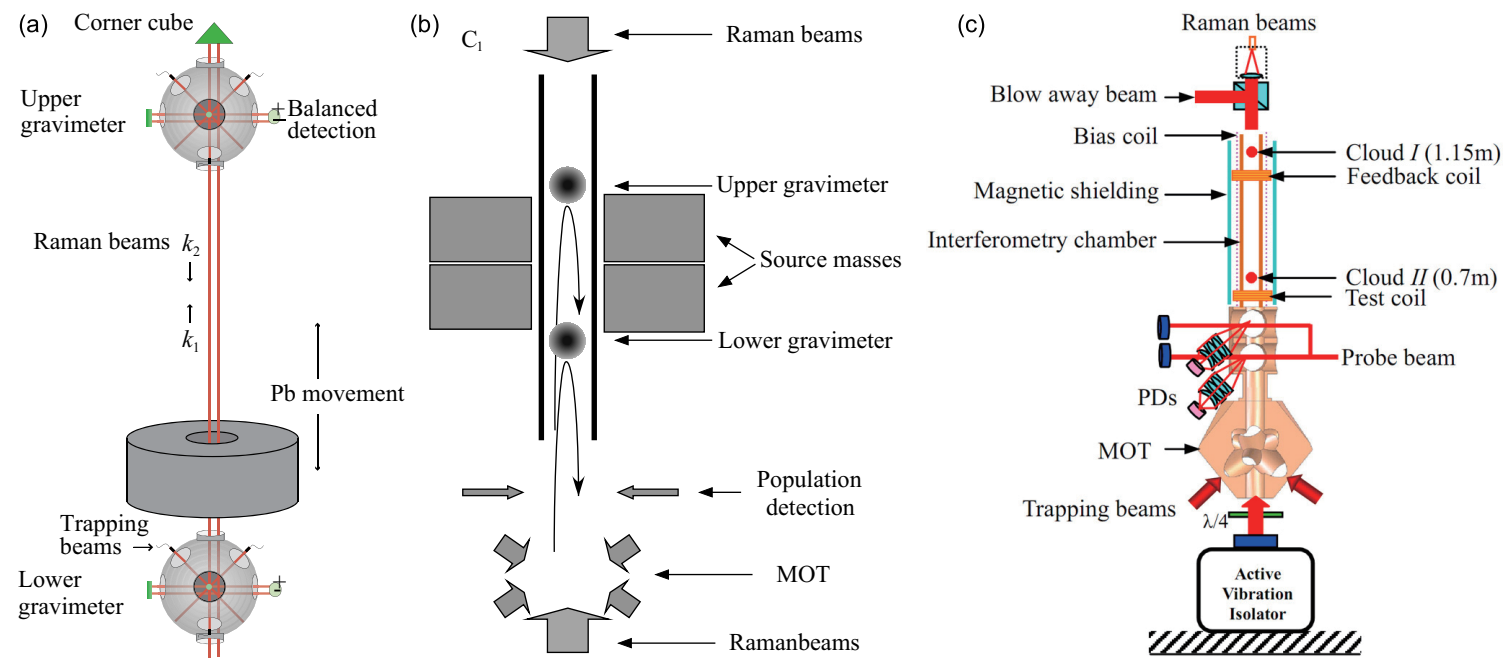

图16 (网络版彩图)原子干涉重力梯度测量. (a) 斯坦福大学Kasevich小组的原子干涉重力梯度仪 ${ }^{[38]}$; (b) 佛罗伦萨大学Tino 组梯度仪 ${ }^{[36]} ;$ (c) 华中科技大学重力梯度仪 ${ }^{[81]}$

Figure 16 (Color online) The measurements of gravity gradient using atomic interferometer. Atom gradiometers at (a) Kasevich's group in Stanford university [38], (b) Tino's group in Firenze University [36] and (c) HUST [81].
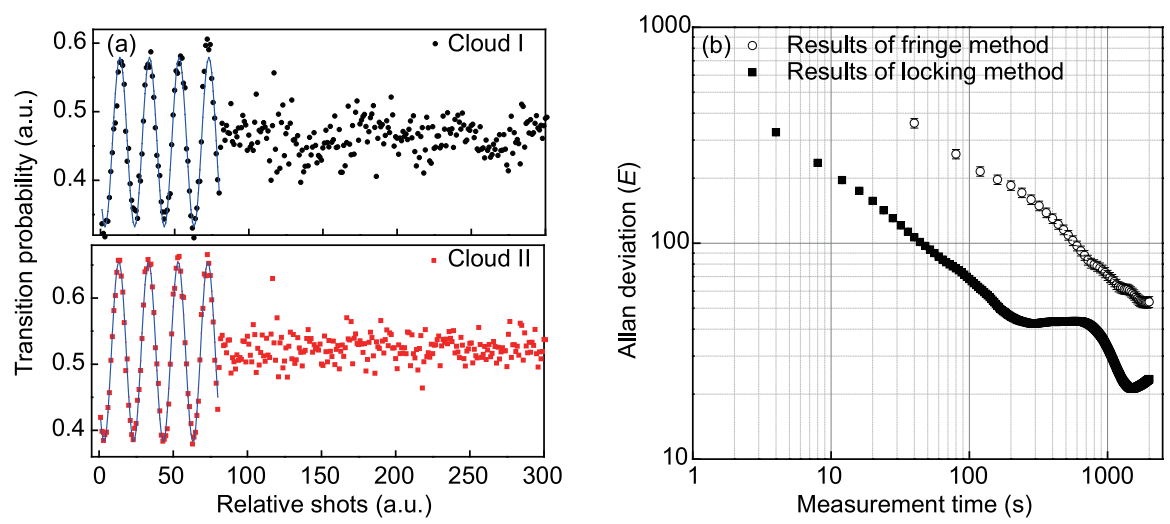

图17 (网络版彩图)(a) 条纹锁定情况下原子干涉重力梯度测量结果; (b) 艾伦方差 ${ }^{[81]}$

Figure 17 (Color online) (a) Results of atom gradiometer with fringe-lock method and (b) the Allan deviation of the results [81]. 
一种新的方法. 传统的万有引力常数测量方法为扭摆

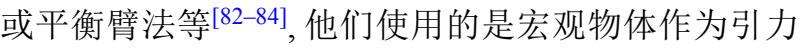
场检验质量, 而且一般需要用扭丝等将摆或者平衡臂 悬挂起来. 原子干涉法的优势在于它使用自由落体的 原子作为检验质量, 避免了扭丝和摆的特性引入的系 统误差引入的系统误差. 利用原子干涉法测 $G$ 的一般 方法是利用吸引质量产生引力场, 利用原子干涉仪测 出产生的引力场, 然后通过比较测量得到的干涉相位 和通过牛顿引力定律公式计算得到的干涉相位, 即可 得出引力常量 $G$ 的值. 目前, 国际上利用原子干涉法测 $G$ 的主要有美国斯坦福大学的Kasevich组和意大利佛 罗伦萨大学的 Tino 组 ${ }^{[40]}$.

Kasevich 组 ${ }^{[38]}$ 采用双磁光阱双喷泉的实验装置, 上下两个磁光阱各自形成同步的两个原子喷泉. 两个 喷泉中的原子同时与同一束拉曼光作用形成共轭的 两个原子干涉仪, 这样构成了一个重力梯度仪, 可以 测量差分重力加速度. 这种差分测量的优势是可以差 分掉地球表面的重力加速度并且抑制掉共模噪声, 包 括地面振动噪声和拉曼光相位噪声. 吸引质量周期性 地上下切换位置, 对这两种配置下的测量结果进行差 分可以抵消背景重力场的影响.

Kasevich组在 2007 年率先完成原子干涉法测 $G$ 实 验, 实验结果为 $G=6.693(27)(21) \times 10^{-11} \mathrm{~m}^{3} \mathrm{~kg}^{-1} \mathrm{~s}^{-2}$. 其 中, 括号中数值分别为 $\mathrm{A}$ 类和 $\mathrm{B}$ 类不确定度. 其合成相 对不确定度为 $5080 \mathrm{ppm}\left(1 \mathrm{ppm}=1 \times 10^{-6}\right)$.

Tino组的原子干涉梯度仪采用单磁光阱双喷泉的 方案, 在同一个磁光阱中囚禁上抛两团原子到不同高 度, 形成原子干涉重力梯度仪. 吸引质量分成上下两 层. 两层吸引质量的位置在两种配置下切换. 两种配 置为近程配置(两层吸引质量距离近)和远程配置(两 层吸引质量距离远). 这种配置方法相对Kasevich组能 够有效增大吸引质量引力场引起的干涉相移信号. 两 种配置下测量的差分加速度进行差分, 可以消除背景 重力场的影响.

Tino组 ${ }^{[39]}$ 在 2008 年给出了他们原子干涉法测 $G$ 的 初步结果, 初步结果 $G$ 的测量值为 $6.667(11)(3) \times 10^{-11}$ $\mathrm{m}^{3} \mathrm{~kg}^{-1} \mathrm{~s}^{-2}$, 总的相对不确定度为 $1710 \mathrm{ppm}$. 通过进一 步的改进, 在2014年他们给出了 $G$ 的高精度测量结果: $G=6.67191(77)(62) \times 10^{-11} \mathrm{~m}^{3} \mathrm{~kg}^{-1} \mathrm{~s}^{-2}$, 相对不确定度为 $148 \mathrm{ppm}$.

本课题组在原子干涉重力测量基础上, 给出了原
子干涉法测 $G$ 的方案模, 拟采用布拉格衍射原子干涉 法在 $100 \mathrm{ppm}$ 精度水平上测量 $G$ 值.

\section{3 弱等效原理检验以及相关基础物理研究}

作为广义相对论的基本假设之一, 爱因斯坦弱等 效原理指出自由落体的运动与其内部结构以及组成 成分无关. 但几乎所有试图统一引力与其他三种基本 相互作用的理论都要求弱等效原理破缺. 所以在实验 中对其精确检验就显得尤为重要. 从最初的比萨斜塔 自由落体实验到如今的地月测距 ${ }^{[85]}$ 以及精密扭秤实 验 ${ }^{[86]}$, 宏观上人们已经在 $10^{-13}$ 的精度水平检验了弱等 效原理的正确性.

利用原子干涉技术操控微观原子来检验弱等效 原理的优势主要有三个方面: 一是原子干涉技术具有 更高的潜在检验精度; 二是原子具有更为精确并且多 样的固有属性, 如质量、组成、内部能态、自旋状态 等;三是微观领域内弱等效原理有着更多可能的破缺 机制[87]. 人们可以通过微观原子与宏观物体的比较来 检验不同尺度上自由下落的一致性,也可以通过比较 不同元素、不同核子数或者不同内态的原子之间的 下落加速度差异来检验弱等效原理是否成立.

斯坦福大学朱棣文小组 ${ }^{[88}$ 在 1999年首次利用原 子干涉仪和角雉型绝对重力仪在 $7 \times 10^{-9}$ 精度上验证了 微观粒子与宏观物体自由下落加速度的一致性. 德国 马克思-普朗克量子光学研究所Hänsch 小组 ${ }^{[89]}$ 在 2004 年利用原子干涉的方法将 ${ }^{85} \mathrm{Rb}$ 与 ${ }^{87} \mathrm{Rb}$ 的自由下落加速 度一致性检验到了 $1.7 \times 10^{-7}$ 的水平, 利用处于不同超 精细能级的 ${ }^{85} \mathrm{Rb}$ 原子将弱等效原理检验到了 $1.2 \times 10^{-7}$ 的量级. 10年后, 法国航天实验室的Bresson小组 ${ }^{[90]}$ 报导了他们利用同时双组份原子干涉仪检验 ${ }^{85} \mathrm{Rb}$ 与 ${ }^{87} \mathrm{Rb}$ 原子的自由下落加速度一致性的结果, 检验精度 为 $3.2 \times 10^{-7}$. 随后, 德国汉诺威大学 Rasel 小组 ${ }^{[91]}$ 利用 ${ }^{87} \mathrm{Rb}$ 和 ${ }^{39} \mathrm{~K}$ 将不同元素原子间的自由下落加速度一致 性检验到了 $5.4 \times 10^{-7}$ 精度. 意大利佛罗伦萨大学 Tino 小组 ${ }^{[92]}$ 利用 ${ }^{88} \mathrm{Sr}$ 玻色子和 ${ }^{87} \mathrm{Sr}$ 费米子将自旋-重力耦 合相关的弱等效原理检验到了 $1.6 \times 10^{-7}$ 精度. 国内的 武汉物理与数学研究所利用 4 波双衍射拉曼跃迁的方 式将 ${ }^{85} \mathrm{Rb}$ 与 ${ }^{87} \mathrm{Rb}$ 的自由下落加速度一致性检验到了 $3.0 \times 10^{-8}$ 的水平 ${ }^{[93]}$, 这也是目前为止利用原子干涉仪 检验弱等效原理的最高精度. 本课题组首次在 $10^{-7}$ 精 度上检验了 ${ }^{87} \mathrm{Rb}$ 原子不同自旋态 $\left(m_{F}= \pm 1\right)$ 之间自由下 
落加速度的一致性 ${ }^{[94]}$. 此外, 为了在更高精度上检验 弱等效原理, 地面的 $10 \mathrm{~m}$ 喷泉计划 ${ }^{[95]}$ 和空间原子干涉 计划 ${ }^{[96]}$ 也都在进行中, 目标检验精度都在 $10^{-15}$ 量级.

除了检验等效原理外, 原子干涉仪还能用于暗能 量的发现. 暗能量是导致宇宙加速膨胀的可能因素之 一. 加州大学伯克利小组利用高真空中球状质量旁的 铯物质波干涉仪为探测暗能量提供了一种可能的测 量方案 ${ }^{[97]}$. 除此之外, 原子干涉技术也用于其他基础 物理的研究中 ${ }^{[98,99]}$.

\section{4 结论}

本文简要介绍了原子干涉仪的发展历程、科学 用途及其实验装置. 原子干涉仪能够高精度地测量与 加速度相关的物理量, 如重力加速度、地球转动、万 有引力常数等. 在原子干涉重力测量方面, 现阶段原
子干涉仪的分辨率已经不是重力测量精度的主要限 制. 系统误差项的评估, 特别是科里奥利力以及波前 畸变的评估是现阶段原子干涉重力测量的主要限制. 科里奥利力效应可以通过调制实验进行评估. 利用表 面质量更好的光学器件能够有效抑制波前畸变效应.

随着冷原子干涉技术的发展, 原子的物质波操控 方法也变得更为多样化. 除了拉曼脉冲的操控方式, 还 有光晶格布拉格衍射等方式, 其中布拉格组合脉冲的 方式已经能使原子波包相干分裂的距离达到 $0.5 \mathrm{~m}^{[100]}$. 这也为原子干涉技术用于其他精密测量领域提供了 更多的途径.

原子干涉重力测量装置正朝着高精度、高准确 性以及可移动、小型化的方向发展, 广泛应用于引力 物理、地球物理、精密测量、资源勘探以及其他科 学研究及应用领域.

\section{参考文献}

1 Cheinet P, Dos Santos F, Petelski T, et al. Cold atom absolute gravimeter for the watt balance. In: 2004 Conference on Precision Electromagnetic Measurements Digest. London: IEEE, 2004. 60-61

2 Müller H, Chiow S, Herrmann S, et al. Atom-interferometry tests of the isotropy of post-Newtonian gravity. Phys Rev Lett, 2008, 100: 031101

3 Chung K Y, Chiow S, Herrmann S, et al. Atom interferometry tests of local Lorentz invariance in gravity and electrodynamics. Phys Rev D, 2009, 80: 016002

4 Torge W, Rapp R H, Cazenave A A, et al. International association of geodesy symposia. Int Assoc Geodesy Symp, 1996, 116: 20-29

5 Pawlowski B. Gravity gradiometry in resource exploration. Leading Edge, 1998, 17: 51-52

6 Niebauer T M, Sasagawa G S, Faller J E, et al. A new generation of absolute gravimeters. Metrologia, 1995, 32: 159-180

7 Okubo S, Yoshida S, Sato T, et al. Verifying the precision of a new generation absolute gravimeter FG5-Comparison with superconducting gravimeters and detection of oceanic loading tide. Geophys Res Lett, 1997, 24: 489-492

$8 \mathrm{Hu}$ Z K, Sun B L, Duan X C, et al. Demonstration of an ultrahigh-sensitivity atom-interferometry absolute gravimeter. Phys Rev A, 2013, 88: 043610

9 Dalibard J, Cohen-tannoudji C. Laser cooling below the Doppler limit by polarization gradients: Simple theoretical models. J Opt Soc Am B, 1989, 6: 2023

10 Lett P D, Watts R N, Westbrook C I, et al. Observation of atoms laser cooled below the Doppler limit. Phys Rev Lett, 1988, 61: 169-172

11 Phillips W D, Prodan J V, Metcalf H J. Laser cooling and electromagnetic trapping of neutral atoms. J Opt Soc Am B, 1985, 2: 1751-1767

12 Chu S, Hollberg L, Bjorkholm J E, et al. Three-dimensional viscous confinement and cooling of atoms by resonance radiation pressure. Phys Rev Lett, 1985, 55: 48-51

13 Chu S. Laser manipulation of atoms and particles. Science, 1991, 253: 861-866

14 Kasevich M, Chu S. Atomic interferometry using stimulated Raman transitions. Phys Rev Lett, 1991, 67: 181-184

15 Peters A, Chung K Y, Chu S. High-precision gravity measurements using atom interferometry. Metrologia, 2001, 38: 25-61

16 Cronin A D, Schmiedmayer J, Pritchard D E. Optics and interferometry with atoms and molecules. Rev Mod Phys, 2009, 81: 1051-1129

17 Schmidt M, Senger A, Hauth M, et al. A mobile high-precision absolute gravimeter based on atom interferometry. Gyroscopy Navig, 2011, 2: $170-177$

18 Le gouët J, Mehlstäubler T E, Kim J, et al. Limits to the sensitivity of a low noise compact atomic gravimeter. Appl Phys B, 2008, 92: 133-144

19 Zhou L, Xiong Z Y, Yang W, et al. Measurement of local gravity via a cold atom interferometer. Chin Phys Lett, 2011, 28: 013701

20 Wu B, Wang Z, Cheng B, et al. The investigation of a mGal-level cold atom gravimeter for field applications. Metrologia, 2014, 51: 452-458 
21 Zhou M K, Hu Z K, Duan X C, et al. Performance of a cold-atom gravimeter with an active vibration isolator. Phys Rev A, 2012, 86: 043630

22 Louchet-chauvet A, Farah T, Bodart Q, et al. The influence of transverse motion within an atomic gravimeter. New J Phys, 2011, 13: 065025

23 Hauth M, Freier C, Schkolnik V, et al. First gravity measurements using the mobile atom interferometer GAIN. Appl Phys B, 2013, 113: 49-55

24 Nyman R A, Varoquaux G, Lienhart F, et al. I.C.E.: A transportable atomic inertial sensor for test in microgravity. Appl Phys B, 2006, 84: 673-681

25 Ferrari G, Poli N, Sorrentino F, et al. Long-lived Bloch oscillations with bosonic Sr atoms and application to gravity measurement at the micrometer scale. Phys Rev Lett, 2006, 97: 60402

26 Poli N, Wang F Y, Tarallo M G, et al. Precision measurement of gravity with cold atoms in an optical lattice and comparison with a classical gravimeter. Phys Rev Lett, 2011, 106: 038501

27 Tarallo M G, Alberti A, Poli N, et al. Delocalization-enhanced Bloch oscillations and driven resonant tunneling in optical lattices for precision force measurements. Phys Rev A, 2012, 86: 033615

28 Gustavson T L, Bouyer P, Kasevich M A. Precision rotation measurements with an atom interferometer gyroscope. Phys Rev Lett, 1997, 78: 2046-2049

29 Lenef A, Hammond T D, Smith E T, et al. Rotation sensing with an atom interferometer. Phys Rev Lett, 1997, 78: 760-763

30 Canuel B, Leduc F, Holleville D, et al. Six-axis inertial sensor using cold-atom interferometry. Phys Rev Lett, 2006, 97 : 10402

31 Dubetsky B, Kasevich M A. Atom interferometer as a selective sensor of rotation or gravity. Phys Rev A, 2006, 74: 023615

32 Chiow S, Kovachy T, Chien H C, et al. 102hk large area atom interferometers. Phys Rev Lett, 2011, 107: 130403

33 Mcguirk J M, Snadden M J, Kasevich M A. Large area light-pulse atom interferometry. Phys Rev Lett, 2000, 85: 4498-4501

34 Snadden M, Mcguirk J, Bouyer P, et al. Measurement of the Earth's gravity gradient with an atom interferometer-based gravity gradiometer. Phys Rev Lett, 1998, 81: 971-974

35 Mcguirk J M, Foster G T, Fixler J B, et al. Sensitive absolute-gravity gradiometry using atom interferometry. Phys Rev A, 2002, 65: 033608

36 Sorrentino F, Bodart Q, Cacciapuoti L, et al. Sensitivity limits of a Raman atom interferometer as a gravity gradiometer. Phys Rev A, 2014, 89: 023607

37 Biedermann G W, Wu X, Deslauriers L, et al. Testing gravity with cold-atom interferometers. Phys Rev A, 2015, 91: 033629

38 Fixler J B, Foster G T, Mcguirk J M, et al. Atom interferometer measurement of the Newtonian constant of gravity. Science, 2007, 315: 74-77

39 Lamporesi G, Bertoldi A, Cacciapuoti L, et al. Determination of the Newtonian gravitational constant using atom interferometry. Phys Rev Lett, 2008, 100: 050801

40 Rosi G, Sorrentino F, Cacciapuoti L, et al. Precision measurement of the Newtonian gravitational constant using cold atoms. Nature, 2014, 510: $518-521$

41 Hu Z K, Duan X C, Zhou M K, et al. Simultaneous differential measurement of a magnetic-field gradient by atom interferometry using double fountains. Phys Rev A, 2011, 84: 013620

42 Zhou M K, Hu Z K, Duan X C, et al. Precisely mapping the magnetic field gradient in vacuum with an atom interferometer. Phys Rev A, 2010, 82: 061602

43 Dimopoulos S, Graham P W, Hogan J M, et al. Testing general relativity with atom interferometry. Phys Rev Lett, 2007, 98 : 111102

44 Weiss D S, Young B C, Chu S. Precision measurement of $\hbar / \mathrm{m}$ Cs based on photon recoil using laser-cooled atoms and atomic interferometry. Appl Phys B, 1994, 59: 217-256

45 Cadoret M, De mirandes E, Cladé P, et al. Combination of Bloch oscillations with a Ramsey-Bordé interferometer: New determination of the fine structure constant. Phys Rev Lett, 2008, 101: 230801

46 Robertson N A. Laser interferometric gravitational wave detectors. Class Quantum Grav, 2000, 17: R19-R40

47 Tino G M, Vetrano F. Is it possible to detect gravitational waves with atom interferometers? Class Quantum Grav, 2007, 24: 2167-2178

48 Graham P W, Hogan J M, Kasevich M A, et al. New method for gravitational wave detection with atomic sensors. Phys Rev Lett, 2013, 110: 171102

49 Yu N, Kohel J M, Kellogg J R, et al. Development of an atom-interferometer gravity gradiometer for gravity measurement from space. Appl Phys B, 2006, 84: 647-652

50 Tino G M, Sorrentino F, Aguilera D, et al. Precision gravity tests with atom interferometry in space. Nucl Phys B-Proc Suppl, 2013, 243-244: 203-217

51 Bordé C J. Theoretical tools for atom optics and interferometry. Comptes Rendus de l'Académie des Sci -Ser IV - Phys, 2001, 2: 509-530

52 Moler K, Weiss D S, Kasevich M, et al. Theoretical analysis of velocity-selective Raman transitions. Phys Rev A, 1992, 45: 342-348

53 Castin Y, Wallis H, Dalibard J. Limit of Doppler cooling. J Opt Soc Am B, 1989, 6: 2046-2057

54 Treutlein P, Chung K Y, Chu S. High-brightness atom source for atomic fountains. Phys Rev A, 2001, 63: 051401 
55 Dieckmann K, Spreeuw R J C, Weidemüller M, et al. Two-dimensional magneto-optical trap as a source of slow atoms. Phys Rev A, 1998, 58: 3891-3895

56 Bodart Q, Merlet S, Malossi N, et al. A cold atom pyramidal gravimeter with a single laser beam. Appl Phys Lett, 2010, 96: 134101

57 Schmidt M, Prevedelli M, Giorgini A, et al. A portable laser system for high-precision atom interferometry experiments. Appl Phys B, 2011, 102: $11-18$

58 Theron F, Carraz O, Renon G, et al. Narrow linewidth single laser source system for onboard atom interferometry. Appl Phys B, 2015, 118: 1-5

59 Wang Q, Wang Z, Fu Z, et al. A compact laser system for the cold atom gravimeter. Opt Commun, 2016, 358: 82-87

60 Mertikas S P, Merlet S, Le gouët J, et al. International association of geodesy symposia. Int Assoc Geodesy Symp, 2010, 135: 115-121

61 Rinker III R L. Super Spring - A New Type of Low-Frequency Vibration Isolator. Dissertation for Doctoral Degree. Boulder: University of Colorado, 1983

62 Hensley J M, Peters A, Chu S. Active low frequency vertical vibration isolation. Rev Sci Instrum, 1999, 70: 2735

63 Zhou M K, Xiong X, Chen L L, et al. Note: A three-dimension active vibration isolator for precision atom gravimeters. Rev Sci Instrum, 2015, 86: 046108

64 Heupel T, Weitz M, Hänsch T W. Phase-coherent light pulses for atom optics and interferometry. Opt Lett, 1997, 22: 1719-1721

65 Le gouët J, Kim J, Bourassin-bouchet C, et al. Wide bandwidth phase-locked diode laser with an intra-cavity electro-optic modulator. Opt Commun, 2009, 282: 977-980

66 Marino A M, Stroud C R. Phase-locked laser system for use in atomic coherence experiments. Rev Sci Instrum, 2008, 79: 013104

67 Yim S H, Lee S B, Kwon T Y, et al. Optical phase locking of two extended-cavity diode lasers with ultra-low phase noise for atom interferometry. Appl Phys B, 2014, 115: 491-495

68 Lee K S, Kim J, Lee S B, et al. A phase-modulated laser system of ultra-low phase noise for compact atom interferometers. J Korean Phys Soc, 2015, 67: 318-322

69 Bidel Y, Carraz O, Charrière R, et al. Compact cold atom gravimeter for field applications. Appl Phys Lett, 2013, 102: 144107

70 Freier C, Hauth M, Schkolnik V, et al.Mobile quantum gravity sensor with unprecedented stability. arxiv:1512.05660v1

71 Gillot P, Francis O, Landragin A, et al. Stability comparison of two absolute gravimeters: optical versus atomic interferometers. Metrologia, 2014, 51: L15

72 Altin P A, Johnsson M T, Negnevitsky V, et al. Precision atomic gravimeter based on Bragg diffraction. New J Phys, 2013, 15: 023009

73 Zhan S, Duan X C, Zhou M K, et al. Investigating the frequency-dependent amplification of a tapered amplifier in atom interferometers. Opt Lett, 2015, 40: 29

74 Cheng B, Gillot P, Merlet S, et al. Influence of chirping the Raman lasers in an atom gravimeter: Phase shifts due to the Raman light shift and to the finite speed of light. Phys Rev A, 2015, 92: 063617

75 Lan S Y, Kuan P C, Estey B, et al. Influence of the coriolis force in atom interferometry. Phys Rev Lett, 2012, 108: 090402

76 Schkolnik V, Leykauf B, Hauth M, et al. The effect of wavefront aberrations in atom interferometry. Appl Phys B, 2015, 120: 311-316

77 Dimopoulos S, Graham P W, Hogan J M, et al. Atomic gravitational wave interferometric sensor. Phys Rev D, 2008, 78: 122002

78 D'agostino G, Merlet S, Landragin A, et al. Perturbations of the local gravity field due to mass distribution on precise measuring instruments: A numerical method applied to a cold atom gravimeter. Metrologia, 2011, 48: 299-305

79 Gillot P, Francis O, Landragin A, et al. Stability comparison of two absolute gravimeters: optical versus atomic interferometers. Metrologia, 2014, 51: L15

80 Clairon A, Laurent P, Santarelli G, et al. A cesium fountain frequency standard: preliminary results. IEEE Trans Instrum Meas, 1995, 44: 128-131

81 Duan X C, Zhou M K, Mao D K, et al. Operating an atom-interferometry-based gravity gradiometer by the dual-fringe-locking method. Phys Rev A, 2014, 90: 023617

82 Luo J, Liu Q, Tu L C, et al. Determination of the Newtonian gravitational constant G with time-of-swing method. Phys Rev Lett, 2009, 102: 240801

83 Gundlach J H, Merkowitz S M. Measurement of Newton's constant using a torsion balance with angular acceleration feedback. Phys Rev Lett, 2000, 85: 2869-2872

84 Michaelis W, Haars H, Augustin R. A new precise determination of Newton's gravitational constant. Metrologia, 1995, 32: 267-276

85 Williams J G, Turyshev S G, Boggs D H. Lunar laser ranging tests of the equivalence principle with the earth and moon. Int J Mod Phys D, 2009, 18: $1129-1175$

86 Schlamminger S, Choi K Y, Wagner T A, et al. Test of the equivalence principle using a rotating torsion balance. Phys Rev Lett, 2008, 100: 041101

87 Lämmerzahl C. Quantum tests of the foundations of general relativity. Class Quantum Grav, 1998, 15: 13-27 
88 Chu S, Peters A, Chung K Y. Measurement of gravitational acceleration by dropping atoms. Nature, 1999, 400: 849-852

89 Fray S, Diez C A, Hänsch T W, et al. Atomic interferometer with amplitude gratings of light and its applications to atom based tests of the equivalence principle. Phys Rev Lett, 2004, 93: 240404

90 Bonnin A, Zahzam N, Bidel Y, et al. Simultaneous dual-species matter-wave accelerometer. Phys Rev A, 2013, 88: 043615

91 Schlippert D, Hartwig J, Albers H, et al. Quantum test of the universality of free fall. Phys Rev Lett, 2014, 112: 203002

92 Tarallo M G, Mazzoni T, Poli N, et al. Test of Einstein equivalence principle for 0-spin and half-integer-spin atoms: Search for spin-gravity coupling effects. Phys Rev Lett, 2014, 113: 023005

93 Zhou L, Long S, Tang B, et al. Test of equivalence principle at $10^{-8}$ level by a dual-species double-diffraction raman atom interferometer. Phys Rev Lett, 2015, 115: 013004

94 Duan X C, Zhou M K, Deng X B, et al.Test of the universality of free fall with atoms in different spin Orientations. arxiv:1503.00433

95 Dickerson S M, Hogan J M, Sugarbaker A, et al. Multiaxis inertial sensing with long-time point source atom interferometry. Phys Rev Lett, 2013, 111: 083001

96 Altschul B, Bailey Q G, Blanchet L, et al. Quantum tests of the Einstein equivalence principle with the STE-QUEST space mission. Adv Space Res, 2015, 55: 501-524

97 Hamilton P, Jaffe M, Haslinger P, et al. Atom-interferometry constraints on dark energy. Science, 2015, 349: 849-851

98 Hamilton P, Zhmoginov A, Robicheaux F, et al. Antimatter interferometry for gravity measurements. Phys Rev Lett, 2014, 112: 121102

99 Rosi G, Cacciapuoti L, Sorrentino F, et al. Measurement of the gravity-field curvature by atom interferometry. Phys Rev Lett, 2015, 114: 013001

100 Kovachy T, Asenbaum P, Overstreet C, et al. Quantum superposition at the half-metre scale. Nature, 2015, 528: 530-533

\title{
Precision gravity measurements with cold atom interferometer
}

\author{
CHEN LeLe, LUO Qin, DENG XiaoBing, TAN YuJie, MAO DeKai, ZHANG Heng, \\ ZHOU MinKang, DUAN XiaoChun, SHAO ChengGang \& HU ZhongKun* \\ Ministry of Education Key Laboratory of Fundamental Physical Quantities Measurements, School of Physics, \\ Huazhong University of Science and Technology, Wuhan 430074, China
}

Cold-atom interferometer based on stimulated Raman transition is interesting for both fundamental researches and precision measurements. Measuring $g$, the free fall acceleration, plays an important role in these applications. We describe the theory of atom interferometry, including the evolution of the atomic wavepacket in gravity field, the laser-atom interaction, and the interference of the atomic waves. In this article, we introduce the procedure of measuring $g$ using atom interferometer, the experimental setup, and the tendency of the future development. The works of the domestic and international groups for $g$ measurement are concluded. Furthermore, the noise limits and dominant systematic effects in atom gravimeters are analyzed. The suppressions of the background vibration, the Raman phase noise, and the evaluation of the main systematic effects, are introduced. Finally, the other applications using the atom interferometers for precision measurement are presented, such as in measuring the Newton's gravitational constant, in testing the weak equivalence principle and the other fundamental applications in physics studies.

matte wave, gravity acceleration, atom interferometer, cold atom

PACS: 03.75.-b, 06.30.Gv, 07.07.Df, 39.25.+k

doi: $10.1360 /$ SSPMA2016-00156 